\title{
Strengthened Ordered Directionally monotone functions. Links between the different notions of monotonicity
}

\begin{abstract}
Mikel Sesma-Sara ${ }^{\mathrm{a}, \mathrm{b}}$, Julio Lafuente ${ }^{\mathrm{a}}$, Antonio Roldán ${ }^{\mathrm{c}, \mathrm{d}}$, Radko Mesiar ${ }^{\mathrm{e}, \mathrm{f}}$, Humberto Bustince ${ }^{\mathrm{a}, \mathrm{b}, *}$

${ }^{a}$ Departamento de Estadística, Informática y Matemáticas, Universidad Pública de Navarra, Campus Arrosadia s/n, 31006, Pamplona, Spain

${ }^{b}$ Institute of Smart Cities, Universidad Pública de Navarra, 31006, Pamplona, Spain

${ }^{c}$ Department of Mathematics Education, University of Granada, Granada, Spain

${ }^{d}$ PAIDI Research Group, FQM-268, University of Jaén, Jaén, Spain

${ }^{e}$ Department of Mathematics and Descriptive Geometry, Faculty of Civil Engineering, Slovak University of Technology, Radlinského 11, Bratislava, Slovakia

${ }^{f}$ Institute for Research and Applications of Fuzzy Modelling, University of Ostrava, 30. dubna 22, Ostrava 1, Czech Republic
\end{abstract}

\begin{abstract}
In this work, we propose a new notion of monotonicity: strengthened ordered directional monotonicity. This generalization of monotonicity is based on directional monotonicity and ordered directional monotonicity, two recent weaker forms of monotonicity. We discuss the relation between those different notions of monotonicity from a theoretical point of view. Additionally, along with the introduction of two families of functions and a study of their connection to the considered monotonicity notions, we define an operation between functions that generalizes the Choquet integral and the Łukasiewicz implication.

Keywords: Strengthened ordered directional monotonicity; Directional monotonicity; Generalizations of monotonicity.

\footnotetext{
* Corresponding author

Email addresses: mikel.sesma@unavarra.es (Mikel Sesma-Sara), lafuente@unavarra.es (Julio Lafuente), afroldan@ujaen.es (Antonio Roldán), mesiar@math.sk (Radko Mesiar), bustince@unavarra.es (Humberto Bustince)
} 


\section{Introduction}

Monotonicity with respect to each argument is one of the axioms around which the concept of aggregation function is built (see 1, 9]). Aggregation functions' goal is to fuse information, generating a representative value from a 5 number of inputs, and these functions are used in a very vast and diverse field of applications [7, 8, 10, 14]. However, the mentioned condition of monotonicity with respect to every argument is sometimes excessively restrictive, which causes to drop from the theoretical framework functions that otherwise are sound for certain applications, such as fuzzy implication operators, the mode, the Gini

10 and Lehmer means, etc. (see [3]).

With the purpose of creating a wider framework of functions that are valid to fuse information, recently some weaker forms of monotonicity have arised (see [2]). In [16], Wilkin and Beliakov introduced the notion of weak monotonicity, which can be seen as monotonicity along the ray $(1,1, \ldots, 1)$. This interpretation along any ray in $\mathbb{R}^{n}$ and originating directional monotonicity [6]. The possibility of choosing any vector $\vec{r}$ allows to pick a function that increases accordingly to the needs of a certain application, with no need of it being increasing with respect to each of its arguments.

However, both of the aforementioned notions require that the direction of increasingness or decreasingness is fixed beforehand and does not vary according to the point of the domain that is being considered. Based on Yager's ideas ([17]), in [5] ordered directionally monotone functions were introduced. This notion of monotonicity enables the direction of increasingness (or decreasingness) to vary from one point to another. Specifically, the direction of monotonicity depends on the relative size of the inputs, provided that a certain comonotonicity condition is fulfilled.

The relaxation of the monotonicity condition for aggregation functions is listed as a recent trend in Aggregation Theory [13. One of the main advances of 30 the introduction of directional monotonicity is the formation of the so called pre- 
aggregation functions [12, which have been successfully applied in fuzzy rulebased classification problems [11. Furthermore, ordered directionally monotone functions have been used in the field of computer vision, see [5, 15] for an application of ordered directionally monotone functions in edge detection.

The restriction that comes from the comonotonicity condition in the definition of ordered directional monotonicity makes us limit to the cases in which the input vector and the result of increasing it along a direction are comonotone, making the family of ordered directionally monotone functions larger than if the condition were removed. This work attempts to achieve the following goals:

- To introduce a new generalization of monotonicity based on ordered directional monotonicity but with no comonotonicity condition.

- To study the properties and relations between the different notions of monotonicity.

- To define two classes of functions and an operation between them that enable to generalize the Choquet integral and the Eukasiewicz implication.

We call the new notion of monotonicity strengthened ordered directional monotonicity. This generalization of monotonicity is based on that of ordered directional monotonicity, but removing the comonotonicity condition from the definition. The family of strengthened ordered directionally monotone functions

${ }_{50}$ is embedded in that of ordered directionally monotone functions, i.e., every strengthened directionally monotone function is ordered directionally monotone, but not the other way around.

Moreover, we carry out a deep study of the properties that the different families of functions satisfy, as well as the relations among them. We show the conditions for which it is equivalent for a function to be increasing with respect to all its arguments and to be increasing in the sense of the discussed different notions of monotonicity.

As to the third goal, we also present two classes of functions - linear fusion functions and ordered linear fusion functions - and show their main properties 
in terms of the distinct types of monotonicity. Additionally, we introduce an operation between functions from $[0,1]^{2}$ to $[0,1]$ that, when applied to ordered linear fusion functions, generalizes the Choquet integral and the Eukasiewicz implication.

This work is organized as follows. In the next section we recall some preliminary notions that are used throughout the paper, including the definition of strengthened ordered directionally monotone functions and some introductory properties. In Section 3 we study a set of properties about the three different notions of monotonicity - directional monotonicity, ordered directional monotonicity and strengthened ordered directional monotonicity - and we show how these concepts are related. In Section 4 we introduce the family of linear fusion functions and the family of ordered linear fusion functions and we show the behaviour of these families of functions in terms of the discussed notions of directional monotonicity. In Section 5 we introduce the operation $*$ between functions for $n=2$ and show how Choquet integrals and the Eukasiewicz implication can be derived from this operation. In Section 6 we present the relation of every notion of monotonicity that is considered throughout the paper and we finish the work with some concluding remarks.

\section{Preliminaries}

Let $n \in \mathbb{N}, n>1$. We use an arrow to refer to vectors of $\mathbb{R}^{n}, \vec{r}=$ so $\left(r_{1}, \ldots, r_{n}\right) \in \mathbb{R}^{n}$ and we set $\vec{r}^{d}=\left(r_{n}, \ldots, r_{1}\right)$.

We use bold letters to specify points of the hypercube $[0,1]^{n}$, so we set $\mathbf{x}=$ $\left(x_{1}, \ldots, x_{n}\right) \in[0,1]^{n}$. In particular, we write $\mathbf{0}=(0, \ldots, 0)$ and $\mathbf{1}=(1, \ldots, 1)$. If $\mathbf{x}, \mathbf{y} \in[0,1]^{n}$, we set $\mathbf{x} \leq \mathbf{y}$ if $x_{i} \leq y_{i}$ for each $i \in\{1, \ldots, n\}$.

$\mathcal{S}_{n}$ denotes the symmetrical group of degree $n$. Given a permutation $\sigma \in$

${ }_{85} \mathcal{S}_{n}$ we denote the inverse permutation by $\sigma^{-1}$, i.e., $\sigma \sigma^{-1}=\mathrm{id}$, and if $\mathbf{a}=$ $\left(a_{1}, \ldots, a_{n}\right)$ and $\sigma \in \mathcal{S}_{n}$, we set $\mathbf{a}_{\sigma}=\left(a_{\sigma(1)}, \ldots, a_{\sigma(n)}\right)$.

If $H \subseteq \mathbb{R}^{n}$, we set $H_{(\geq)}=\left\{\left(h_{1}, \ldots, h_{n}\right) \in H \mid h_{1} \geq \cdots \geq h_{n}\right\}$ (we occasionally refer to $H_{(\leq)}, H_{(>)}, H_{(<)}, H_{(=)}$with the obvious meanings). 
Let us recall the concepts of directional monotonicity and ordered directional

90

Definition $2.1([6])$. Let $F:[0,1]^{n} \rightarrow[0,1]$ and $\vec{r} \in \mathbb{R}^{n}$, we say that $F$ is $\vec{r}$-increasing (resp. $\vec{r}$-decreasing), if for all $c>0$ and $\mathbf{x} \in[0,1]^{n}$ such that $\mathbf{x}+c \vec{r} \in[0,1]^{n}$ it holds that $F(\mathbf{x}+c \vec{r}) \geq F(\mathbf{x})($ resp. $F(\mathbf{x}+c \vec{r}) \leq F(\mathbf{x}))$. If $F(\mathbf{x}+c \vec{r})=F(\mathbf{x})$, then we say that $F$ is $\vec{r}$-constant.

95 Definition $2.2\left([5)\right.$. Let $F:[0,1]^{n} \rightarrow[0,1]$ and $\vec{r} \in \mathbb{R}^{n}$, we say that $F$ is ordered directionally (OD) $\vec{r}$-increasing (resp. OD $\vec{r}$-decreasing) if for all $\mathbf{x} \in$ $[0,1]^{n}, \sigma \in \mathcal{S}_{n}$ and $c>0$ such that $\mathbf{x}_{\sigma} \in[0,1]_{(\geq)}^{n}$ and $\mathbf{x}_{\sigma}+c \vec{r} \in[0,1]_{(\geq)}^{n}$, it holds that $F\left(\mathbf{x}+c \vec{r}_{\sigma^{-1}}\right) \geq F(\mathbf{x})\left(\right.$ resp. $\left.F\left(\mathbf{x}+c \vec{r}_{\sigma^{-1}}\right) \leq F(\mathbf{x})\right)$. If $F\left(\mathbf{x}+c \vec{r}_{\sigma^{-1}}\right)=F(\mathbf{x})$, then we say that $F$ is OD $\vec{r}$-constant. directional monotonicity.

Definition 2.3. Let $F:[0,1]^{n} \rightarrow[0,1]$ and $\vec{r} \in \mathbb{R}^{n}$, we say that $F$ is strengthened ordered directionally (SOD) $\vec{r}$-increasing (resp. SOD $\vec{r}$-decreasing) if for all $\mathbf{x} \in[0,1]^{n}, \sigma \in \mathcal{S}_{n}$ and $c>0$ such that $\mathbf{x}_{\sigma} \in[0,1]_{(\geq)}^{n}$ and $\mathbf{x}_{\sigma}+c \vec{r} \in[0,1]^{n}$, it 105 holds that $F\left(\mathbf{x}+c \vec{r}_{\sigma^{-1}}\right) \geq F(\mathbf{x})$ (resp. $\left.F\left(\mathbf{x}+c \vec{r}_{\sigma^{-1}}\right) \leq F(\mathbf{x})\right)$. If $F\left(\mathbf{x}+c \vec{r}_{\sigma^{-1}}\right)=$ $F(\mathbf{x})$, then we say that $F$ is SOD $\vec{r}$-constant.

For brevity, to refer to a function $F$ that is monotone according to each of the defined types, we say that $F$ is $\mathrm{T} \vec{r}$-increasing for $\mathrm{T} \in\{\emptyset$, SOD, OD $\}$. Moreover, we say that $F$ is $\mathrm{T} \vec{r}$-monotone if it is either $\mathrm{T} \vec{r}$-increasing or $\mathrm{T}$ $\vec{r}$-decreasing for $\mathrm{T} \in\{\emptyset$, SOD, OD $\}$.

Note that the case in which $\vec{r}=\overrightarrow{0}$ is trivial, as every function is $\mathrm{T} \overrightarrow{0}-$ increasing, $\mathrm{T} \overrightarrow{0}$-decreasing and $\mathrm{T} \overrightarrow{0}$-constant, for $\mathrm{T} \in\{\emptyset$, SOD, OD $\}$.

Of course SOD $\vec{r}$-increasingness implies OD $\vec{r}$-increasingness and we will see that the reciprocal statement is not true in general. A first flash of the differences between these classes of monotonicity can be done by the observation of the points in $[0,1]^{n}$ for which a function $F:[0,1]^{n} \rightarrow[0,1]$ trivially satisfies the conditions from the different classes of monotonicity. We refer to these points as special.

For instance, for a function $F:[0,1]^{n} \rightarrow[0,1]$ to be increasing (if $\mathbf{x}, \mathbf{y} \in$ 120 $[0,1]^{n}$ such that $\mathbf{x} \leq \mathbf{y}$, then $\left.F(\mathbf{x}) \leq F(\mathbf{y})\right)$, the unique special point is $\mathbf{1}$, as with $\mathbf{x}=\mathbf{1}$ only the trivial situation $\mathbf{y}=\mathbf{1}$ is to be considered (and if $F$ is in fact increasing, then $\left.F(\mathbf{1})=\max \left\{F(\mathbf{x}) \mid \mathbf{x} \in[0,1]^{n}\right\}\right)$. 
The conditions that characterize the special points $\mathbf{x} \in[0,1]^{n}$ for the considered types of increasingness, associated to vectors $\vec{r} \in \mathbb{R}^{n}$, appear in Table 1 .

Table 1: Conditions that characterize the specials points $\mathbf{x} \in[0,1]^{n}$ for each type of increasingness.

\begin{tabular}{|c|c|}
\hline$F$ & if $0<c \in \mathbb{R}, \sigma \in \mathcal{S}_{n}$, then \\
\hline \hline$\vec{r}$-increasing & $\mathbf{x}+c \vec{r} \notin[0,1]^{n}$ \\
SOD $\vec{r}$-increasing & $\mathbf{x}_{\sigma} \in[0,1]_{(\geq)}^{n} \Longrightarrow \mathbf{x}_{\sigma}+c \vec{r} \notin[0,1]^{n}$ \\
OD $\vec{r}$-increasing & $\mathbf{x}_{\sigma} \in[0,1]_{(\geq)}^{n} \Longrightarrow \mathbf{x}_{\sigma}+c \vec{r} \notin[0,1]_{(\geq)}^{n}$ \\
\hline
\end{tabular}

Set $O=(0,0), X_{1}=(1,0), X_{2}=(0,1)$ and $U=(1,1)$. In Figure 1 we see an example of a special point $\mathbf{x}$ for $\vec{r}$-increasingness.

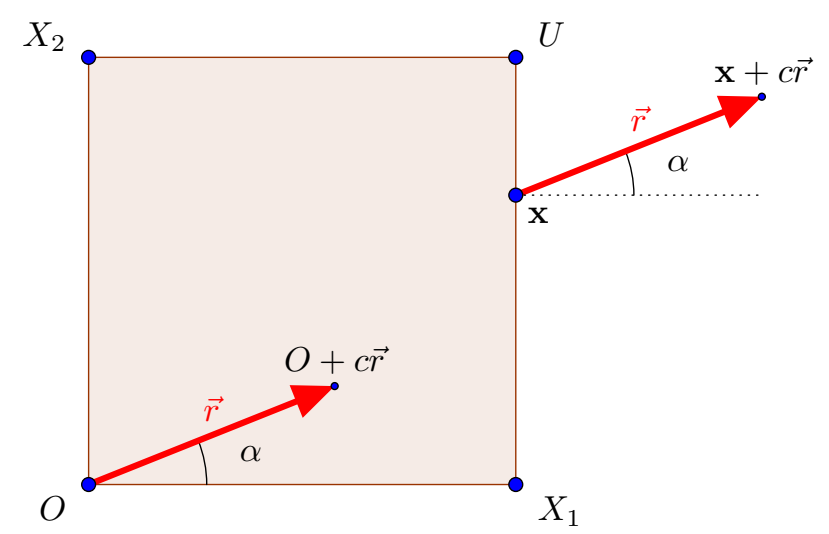

Figure 1: Example of special point $\mathbf{x} \in[0,1]^{2}$ for a direction $\vec{r} \in \mathbb{R}^{2}$.

It is clear that for each $\overrightarrow{0} \neq \vec{r} \in \mathbb{R}^{n}$ whose angle $\alpha$ with $O X_{1}$ satisfies $\alpha \in$ $(0, \pi / 2)$ the corresponding special points form the join of the closed segments ${ }_{130} \quad X_{2} U$ and $U X_{1}$. We illustrate this situation in Figure 2 . 


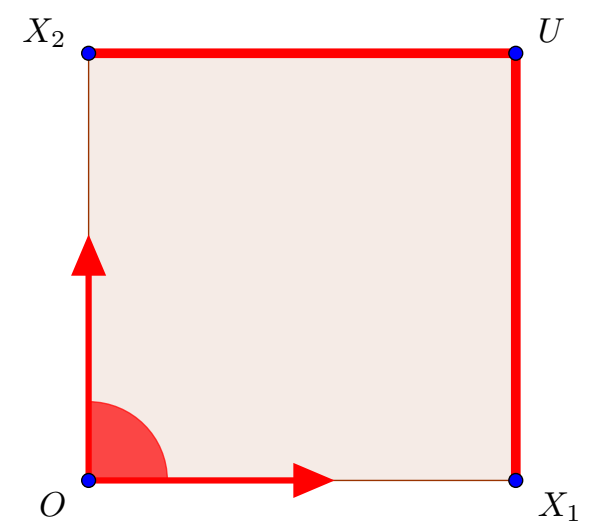

Figure 2: Directions $\overrightarrow{0} \neq \vec{r} \in \mathbb{R}^{2}$ whose angle with $O X_{1}$ satisfies $\alpha \in(0, \pi / 2)$ and their corresponding special points; the join of the closed segments $X_{2} U$ and $U X_{1}$.

If $\alpha=0$ however only the points of the closed segment $U X_{1}$ are special, and for $\alpha \in(3 \pi / 2,2 \pi)$, the special points form the joint of the closed segments $O X_{1}$ and $U X_{1}$. In this sense, $\alpha=0$ marks a transition (as also do $\alpha=\pi / 2, \alpha=\pi$ and $\alpha=3 \pi / 2)$.

We depict the different situations by means of schemes which associate, through the use of colors, vectors of transition and corresponding special points in the case $n=2$. For a vector $\vec{r}$ lying between two consecutive transition vectors, the set of special points is the join of the special points corresponding to the consecutive transition vectors. Figure 3 shows the transition vectors and sets of special points for the case of directional monotonicity and Figures 4 and 5 for the cases of SOD monotonicity and OD monotonicity, respectively. 

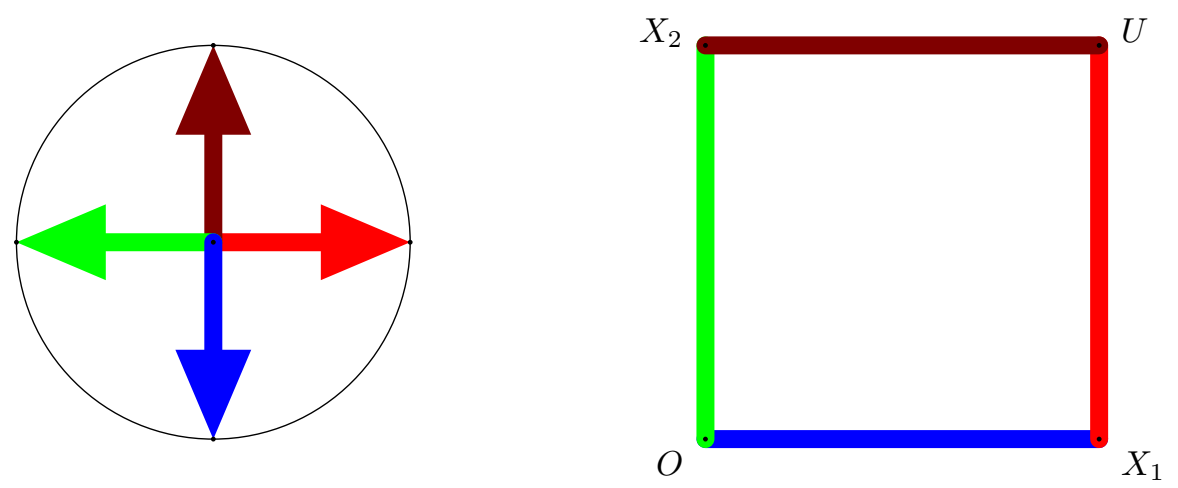

Figure 3: Left: Transition vectors for directional monotonicity. Right: Sets of special points in $[0,1]^{2}$ for directional monotonicity.
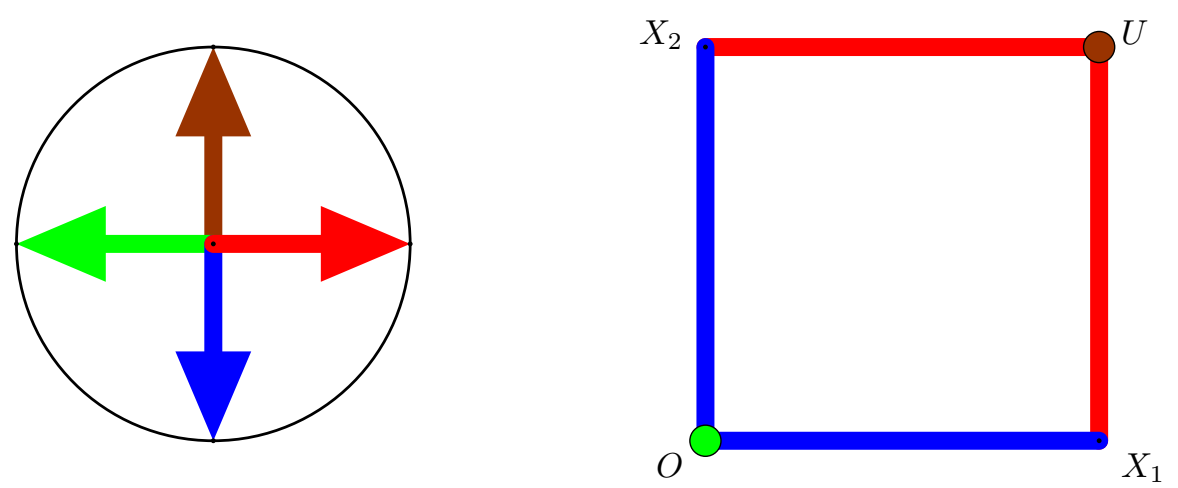

Figure 4: Left: Transition vectors for SOD monotonicity. Right: Sets of special points in $[0,1]^{2}$ for SOD monotonicity.

Observe, for instance, the case of Figure 4 in which $F$ is SOD $\vec{r}$-increasing. For $\alpha=\pi / 2$ the only special point is $U$ and for $\alpha=\pi$ the only one is $O$. If $\alpha \in(3 \pi / 2,2 \pi)$, then the special points draw the perimeter of the square $O X_{1} U X_{2}$. If $\alpha \in(\pi / 2, \pi)$, only the points $O$ and $U$ are special. 

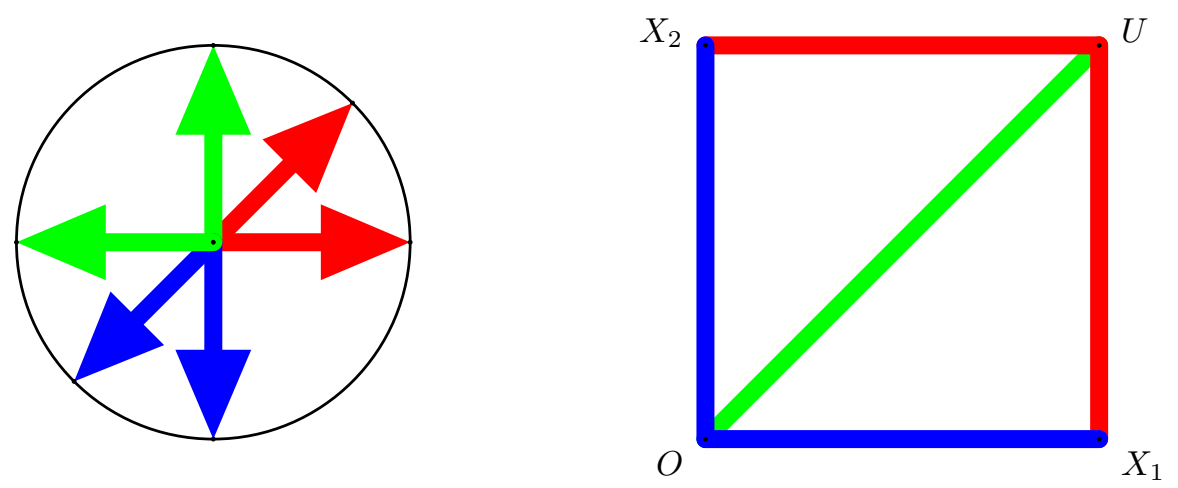

Figure 5: Left: Transition vectors for OD monotonicity. Right: Sets of special points in $[0,1]^{2}$ for OD monotonicity.

In the case of Figure 5 in which $F$ is OD $\vec{r}$-increasing, for $\pi / 2 \leq \alpha \leq \pi$ the special points form the diagonal $O U$.

Remark 2.4. An obvious generalization of the introduced concepts of (S)OD $\vec{r}$-increasingness appears changing in the definitions the triangle $[0,1]_{(\geq)}^{n}$ for any subset $\mathbf{S}$ of $[0,1]^{n}$.

\section{Basic facts}

We begin by developing some basic properties on $\mathrm{T} \vec{r}$-monotonicity for $\mathrm{T} \in$ $\{\emptyset, \mathrm{SOD}, \mathrm{OD}\}$.

First of all we remark that in the definitions corresponding to SOD and $\mathrm{OD}$, instead of having required that $\mathbf{x}_{\sigma} \in[0,1]_{(\geq)}^{n}$, we could set $\mathbf{x}_{\sigma} \in[0,1]_{(\leq)}^{n}$. The corresponding developments would be equivalent, as the following remark states for the OD case (the SOD case is similar).

Proposition 3.1. For all $\mathbf{x} \in[0,1]^{n}, \vec{r} \in \mathbb{R}^{n}, 0<c \in \mathbb{R}$ and $\sigma \in \mathcal{S}_{n}$ the following assertions are equivalent:

(1) $\mathbf{x}_{\sigma}, \mathbf{x}_{\sigma}+c \vec{r} \in[0,1]_{(\geq)}^{n} \Longrightarrow F\left(\mathbf{x}+c \vec{r}_{\sigma^{-1}}\right) \geq F(\mathbf{x})$.

(2) $\mathbf{x}_{\sigma}, \mathbf{x}_{\sigma}+c \vec{r}^{d} \in[0,1]_{(\leq)}^{n} \Longrightarrow F\left(\mathbf{x}+c\left(\vec{r}^{d}\right)_{\sigma^{-1}}\right) \geq F(\mathbf{x})$.

Proof. We must deal with permutations and in this case it is useful to handle permutation matrices. For $\sigma \in \mathcal{S}_{n}$, the permutation matrix $P_{\sigma}$ denotes the $n \times n$ 
matrix resulting from the application of the permutation $\sigma$ to the indices of the rows of the identity matrix $I_{n}$. With this it is immediate that

$$
\mathbf{x}_{\sigma}=\left(x_{\sigma(1)}, \ldots, x_{\sigma(n)}\right)=\left(x_{1}, \ldots, x_{n}\right) P_{\sigma}=\mathbf{x} P_{\sigma} .
$$

With the permutation matrix $D=\left(\begin{array}{cccc}0 & \cdots & 0 & 1 \\ 0 & \cdots & 1 & 0 \\ \vdots & . \cdot & \vdots & \vdots \\ 1 & \cdots & 0 & 0\end{array}\right)$, we have $\vec{r}^{d}=\vec{r} D$.

Let us show that $(1) \Longrightarrow(2)$. Assume that $\mathbf{x}_{\sigma}, \mathbf{x}_{\sigma}+c \vec{r}^{d} \in[0,1]_{(\leq)}^{n}$, that is, $\mathbf{x} P_{\sigma}, \mathbf{x} P_{\sigma}+c \vec{r} D \in[0,1]_{(\leq)}^{n}$. Then obviously $\mathbf{x} P_{\sigma} D, \mathbf{x} P_{\sigma} D+c \vec{r} \in[0,1]_{(\geq)}^{n}$, as $D^{2}=I_{n}$. Then, by $(1), F\left(\mathbf{x}+c \vec{r}\left(P_{\sigma} D\right)^{-1}\right) \geq F(\mathbf{x})$. As $\left(P_{\sigma} D\right)^{-1}=D^{-1} P_{\sigma}^{-1}=$ $D P_{\sigma^{-1}}$, and $\vec{r} D P_{\sigma^{-1}}=\vec{r}^{d} P_{\sigma^{-1}}=\left(\vec{r}^{d}\right)_{\sigma^{-1}}$, we have the thesis. Analogously one shows that $(2) \Longrightarrow(1)$.

Let $F:[0,1]^{n} \rightarrow[0,1]$ and let us set, for $\mathrm{T} \in\{\emptyset$, SOD, OD $\}$, the notation

$$
\begin{aligned}
\mathcal{C}_{\mathrm{T}}(F) & =\left\{\vec{r} \in \mathbb{R}^{n} \mid F \text { is } \mathrm{T} \vec{r} \text {-constant }\right\} \\
\mathcal{D}_{\mathrm{T}}^{\uparrow}(F) & =\left\{\vec{r} \in \mathbb{R}^{n} \mid F \text { is } \mathrm{T} \vec{r} \text {-increasing }\right\}
\end{aligned}
$$

particularizing $\mathcal{C}(F)=\mathcal{C}_{\emptyset}(F)$ and $\mathcal{D}^{\uparrow}(F)=\mathcal{D}_{\emptyset}^{\uparrow}(F)$.

We next result follows immediately.

Proposition 3.2. Let $F:[0,1]^{n} \rightarrow[0,1]$ be a function. Then

(1) $\mathcal{C}_{T}(F) \subseteq \mathcal{D}_{T}^{\uparrow}(F)$ for $T \in\{\emptyset, S O D, O D\}$.

(2) $\mathcal{C}_{S O D}(F) \subseteq \mathcal{C}_{O D}(F)$

(3) $\mathcal{D}_{S O D}^{\uparrow}(F) \subseteq \mathcal{D}_{O D}^{\uparrow}(F)$.

Proposition 3.3. If $\vec{r} \in \mathbb{R}_{(\geq)}^{n}$, then $F$ is $S O D \vec{r}$-increasing if and only if $F$ is

Observe that the statements of Propositions 3.2 and 3.3 changing increasingness by decreasingness are valid.

Proposition 3.4. Let $F:[0,1]^{n} \rightarrow[0,1]$ be a function and $k>0$.

(1) For $T \in\{\emptyset, S O D, O D\}, F$ is simultaneously $T \vec{r}$-increasing and $T \vec{r}$ decreasing if and only if $F$ is $T \vec{r}$-constant.

(2) For $T \in\{\emptyset, S O D, O D\}, F$ is $T \vec{r}$-increasing (resp. decreasing) if and only if $F$ is $T(k \vec{r})$-increasing (resp. decreasing). 
(3) For $T \in\{\emptyset, O D\}, F$ is $T \vec{r}$-increasing if and only if $F$ is $T(-k \vec{r})$ decreasing.

Proof. (1) and (2) They are immediate.

(3) By (2) we may assume that $k=1$. Suppose that $F$ is OD $\vec{r}$-increasing. Let $\mathbf{x} \in[0,1]^{n}, k>0$ and $\sigma \in \mathcal{S}_{n}$ such that $\mathbf{x}_{\sigma}, \mathbf{x}_{\sigma}+k(-\vec{r}) \in[0,1]_{(\geq)}^{n}$. Set $\mathbf{y}=$ $\mathbf{x}+k(-\vec{r})_{\sigma^{-1}}$. We have that $\mathbf{y}_{\sigma}=\mathbf{x}_{\sigma}+k(-\vec{r}) \in[0,1]_{(\geq)}^{n}\left(\right.$ hence $\left.\mathbf{y} \in[0,1]^{n}\right)$ and $\mathbf{y}_{\sigma}+k \vec{r}=\mathbf{x}_{\sigma} \in[0,1]_{(\geq)}^{n}$. As $F$ is OD $\vec{r}$-increasing, we have $F(\mathbf{y}) \leq F\left(\mathbf{y}+k \vec{r}_{\sigma^{-1}}\right)$, that is $F\left(\mathbf{x}+k(-\vec{r})_{\sigma^{-1}}\right) \leq F(\mathbf{x})$, hence $F$ is OD $(-\vec{r})$-decreasing. Analogously one has the converse statement.

Similarly one shows that $F$ is $\vec{r}$-increasing if and only if it is $(-k \vec{r})$-decreasing.

Item (2) of Proposition 3.4 shows that the vectors used for determining directions in all considered cases $\mathrm{T} \in\{\emptyset$, SOD, OD $\}$ can be normalized. Item 3 shows that for $\mathrm{T} \in\{\emptyset, \mathrm{OD}\}$, the developments which result by considering $\mathrm{T}$ increasingness and decreasingness are equivalent. However, in Section 5 we show that, in general, this is false for $T=S O D$.

Proposition 3.5. Let $F:[0,1]^{n} \rightarrow[0,1]$ and $\vec{r} \in \mathbb{R}^{n}$. Let $F^{c}:[0,1]^{n} \rightarrow[0,1]$

(1) For $T \in\{\emptyset, S O D, O D\}$ :

(a) $F$ is $T \vec{r}$-increasing if and only if $F^{c}$ is $T \vec{r}$-decreasing.

(b) $\mathcal{C}_{T}(F)=\mathcal{C}_{T}\left(F^{c}\right)$.

(c) $F$ is $T \vec{r}$-constant if and only if both $F$ and $F^{c}$ are $T \vec{r}$-increasing.

(2) For $T \in\{\emptyset, O D\}, F$ is $T \vec{r}$-increasing if and only if $F^{c}$ is $T(-\vec{r})$-increasing.

Proof. (1) The claims in (a) are direct. For instance, for $\mathrm{T}=\emptyset$, if $c \in \mathbb{R}^{+}$and $\mathbf{x}, \mathbf{x}+c \vec{r} \in[0,1]^{n}$, then $F(\mathbf{x}) \leq F(\mathbf{x}+c \vec{r})$ if and only if $F^{c}(\mathbf{x})=1-F(\mathbf{x}) \geq$ $1-F(\mathbf{x}+c \vec{r})=F^{c}(\mathbf{x}+c \vec{r})$.

(b) and $(c)$ follow from $(a)$ as $\left(F^{c}\right)^{c}=F$.

(2) $F$ is $\mathrm{T} \vec{r}$-increasing if and only if $F$ is $\mathrm{T}(-\vec{r})$-decreasing by Proposition 3.4 and this is equivalent to $F^{c}$ being $\mathrm{T}(-\vec{r})$-increasing by (1).

In Section 5 we show that Proposition 3.5 (2) is in general not true for $T=$ $S O D$.

Proposition 3.6. Let $F:[0,1]^{n} \rightarrow[0,1]$ and $\vec{r} \in \mathbb{R}^{n}$. Let $G:[0,1]^{n} \rightarrow[0,1]$ 215 defined by $G(\mathbf{x})=F(\mathbf{1}-\mathbf{x})$.

(1) $F$ is $\vec{r}$-increasing if and only if $G$ is $(-\vec{r})$-increasing. 
(2) For $T \in\{S O D, O D\}, F$ is $T \vec{r}$-increasing if and only if $G$ is $T(-\vec{r})^{d}$ increasing.

Proof. (1) It is straightforward.

(2) Let $F$ be OD $\vec{r}$-increasing and let $\mathbf{x} \in[0,1]^{n}$. Consider $\sigma \in \mathcal{S}_{n}$ such that $\mathbf{x}_{\sigma} \in[0,1]_{(\geq)}^{n}$ and $c>0$ such that $\mathbf{x}_{\sigma}-c \vec{r}^{d} \in[0,1]_{(\geq)}^{n}$.

Put $\mathbf{y}=\mathbf{1}-\mathbf{x}$. Then $\sigma^{d} \in \mathcal{S}_{n}$, given by $\sigma^{d}(i)=\sigma(n-i+1)$ for all $i=1, \ldots, n$, is a permutation such that $\mathbf{y}_{\sigma^{d}} \in[0,1]_{(\geq)}^{n}$ and $\mathbf{y}_{\sigma^{d}}+c \vec{r} \in[0,1]_{(\geq)}^{n}$. Due to the OD $\vec{r}$-increasingness of $F$, we have $F\left(\mathbf{y}+c \vec{r}_{\left(\sigma^{d}\right)^{-1}}\right) \geq F(\mathbf{y})$, and from $225 \sigma^{d}(i)=\sigma(n-i+1)=k$ we have $\left(\sigma^{d}\right)^{-1}(k)=i$ and $\sigma^{-1}(k)=n-i+1$, hence $\left(\sigma^{d}\right)^{-1}(k)=n-\sigma^{-1}(k)+1$, and finally, $\left(\vec{r}^{d}\right)_{\sigma^{-1}}=\vec{r}_{\left(\sigma^{d}\right)^{-1}}$.

Therefore, we get

$$
\begin{aligned}
G\left(\mathbf{x}+c\left(-\vec{r}^{d}\right)_{\sigma^{-1}}\right) & =F\left(\mathbf{1}-\mathbf{x}+c \vec{r}_{\sigma^{-1}}^{d}\right) \\
& =F\left(\mathbf{y}+c \vec{r}_{\left(\sigma^{d}\right)^{-1}}\right) \\
& \geq F(\mathbf{y})=F(\mathbf{1}-\mathbf{x})=G(\mathbf{x}),
\end{aligned}
$$

which means that $G$ is OD $(-\vec{r})^{d}$-increasing.

The converse follows from the fact that $-\left(-\vec{r}^{d}\right)^{d}=\vec{r}$.

The case of $\mathrm{T}=\mathrm{SOD}$ is analogous.

The dual function $F^{d}$ of a function $F:[0,1]^{n} \rightarrow[0,1]$ is defined for each $\mathbf{x} \in[0,1]^{n}$ by $F^{d}(\mathbf{x})=1-F(\mathbf{1}-\mathbf{x})$. Propositions 3.5 and 3.6 arise the following result.

235 Proposition 3.7. Let $F:[0,1]^{n} \rightarrow[0,1], \vec{r} \in \mathbb{R}^{n}$ and $F^{d}:[0,1]^{n} \rightarrow[0,1]$ be the dual function of $F$ defined by $F^{d}(\mathbf{x})=1-F(\mathbf{1}-\mathbf{x})$.

(1) $F$ is $\vec{r}$-increasing if and only if $F^{d}$ is $\vec{r}$-increasing.

(2) $F$ is $O D \vec{r}$-increasing if and only if $F^{d}$ is $O D \vec{r}^{d}$-increasing.

(3) $F$ is $S O D \vec{r}$-increasing if and only if $F^{d}$ is $S O D-\vec{r}^{d}$-decreasing.

The next result follows from the definition of each notion of monotonicity.

Proposition 3.8. Let $F:[0,1]^{n} \rightarrow[0,1]$ be a $T \vec{r}$-monotone function for $T \in$ $\{\emptyset, S O D, O D\}$. Then, if $\varphi:[0,1] \rightarrow[0,1]$ is an increasing (resp. decreasing) function, then the function $\varphi \circ F:[0,1]^{n} \rightarrow[0,1]$ is an $T \vec{r}$-monotone function of the same (resp. reversed) type as $F$.

245 Lemma 3.9. Assume that the function $F:[0,1]^{n} \rightarrow[0,1]$ satisfies $F\left(\mathbf{x}_{\sigma}\right)=$ $F(\mathbf{x})$ for all $\mathbf{x} \in[0,1]^{n}$ and $\sigma \in \mathcal{S}_{n}$. Then 
(1) $\mathcal{D}^{\uparrow}(F) \subseteq \mathcal{D}_{S O D}^{\uparrow}(F)$ and $\mathcal{C}(F) \subseteq \mathcal{C}_{S O D}(F)$.

(2) $\vec{r} \in \mathcal{D}^{\uparrow}(F)$ if and only if $\vec{r}_{\sigma} \in \mathcal{D}^{\uparrow}(F) \forall \sigma \in \mathcal{S}_{n}$. Analogously for $\mathcal{C}(F)$.

Proof. (1) Let $\vec{r} \in \mathbb{R}^{n}, \mathbf{x} \in[0,1]^{n}, \sigma \in \mathcal{S}_{n}, c \in \mathbb{R}^{+}$with $\mathbf{x}_{\sigma} \in[0,1]_{(\geq)}^{n}, \mathbf{x}_{\sigma}+c \vec{r} \in$ $[0,1]^{n}$. Assume that $F$ is $\vec{r}$-increasing. Then

$$
\begin{aligned}
F\left(\mathbf{x}+c \vec{r}_{\sigma^{-1}}\right) & =F\left(\mathbf{x}_{\sigma}+c \vec{r}\right)\left(\text { as }\left(\mathbf{x}+c \vec{r}_{\sigma^{-1}}\right)_{\sigma}=\mathbf{x}_{\sigma}+c \vec{r}\right) \\
& \geq F\left(\mathbf{x}_{\sigma}\right)(\text { as } F \text { is } \vec{r} \text {-increasing }) \\
& =F(\mathbf{x}),
\end{aligned}
$$

hence $F$ is SOD $\vec{r}$-increasing. Analogously one has $\mathcal{C}(F) \subseteq \mathcal{C}_{\mathrm{SOD}}(F)$.

(2) Let $\vec{r} \in \mathcal{D}^{\uparrow}(F)$. If $\mathbf{x}, \mathbf{x}+c \vec{r}_{\sigma} \in[0,1]^{n}$, then $\mathbf{x}_{\sigma^{-1}}, \mathbf{x}_{\sigma^{-1}}+c \vec{r} \in[0,1]^{n}$, hence $F(\mathbf{x})=F\left(\mathbf{x}_{\sigma^{-1}}\right) \leq F\left(\mathbf{x}_{\sigma^{-1}}+c \vec{r}\right)=F\left(\mathbf{x}+c \vec{r}_{\sigma}\right)$ and so $\vec{r}_{\sigma} \in \mathcal{D}^{\uparrow}(F)$. Analogously for $\mathcal{C}(F)$.

Proposition 3.10. Let $F:[0,1]^{n} \rightarrow[0,1]$ be a function and define the function $\widehat{F}:[0,1]^{n} \rightarrow[0,1]$ as follows: if $\mathbf{x} \in[0,1]^{n}$, take $\sigma \in \mathcal{S}_{n}$ such that $\mathbf{x}_{\sigma} \in[0,1]_{(\geq)}^{n}$ and put $\widehat{F}(\mathbf{x})=F\left(\mathbf{x}_{\sigma}\right)$. If $\vec{r} \in \mathbb{R}^{n}$ is such that $F$ is $\vec{r}$-increasing, then $\widehat{F}$ is $O D$ $\vec{r}$-increasing.

Proof. Let $\mathbf{x} \in[0,1]^{n}, \sigma \in \mathcal{S}_{n}$ and $c \in \mathbb{R}^{+}$such that $\mathbf{x}_{\sigma}, \mathbf{x}_{\sigma}+c \vec{r} \in[0,1]_{(\geq)}^{n}$. Then, with $\mathbf{y}=\mathbf{x}+c \vec{r}_{\sigma^{-1}}$, we have that $\mathbf{y}_{\sigma}=\mathbf{x}_{\sigma}+c \vec{r} \in[0,1]_{(\geq)}^{n}$. So, by definition of $\widehat{F}$,

$$
\widehat{F}\left(\mathbf{x}+c \vec{r}_{\sigma^{-1}}\right)=\widehat{F}(\mathbf{y})=F\left(\mathbf{y}_{\sigma}\right)=F\left(\mathbf{x}_{\sigma}+c \vec{r}\right) \geq F\left(\mathbf{x}_{\sigma}\right)=\widehat{F}(\mathbf{x}),
$$

as $F$ is $\vec{r}$-increasing.

In Section 5 we show that in the hypothesis of Proposition $3.10, \widehat{F}$ is not necessarily SOD $\vec{r}$-increasing.

Remark 3.11. Consider a function $F:[0,1]^{n} \rightarrow[0,1]$. Then

a. $\widehat{(\widehat{F})}=\widehat{F}$.

b. $\widehat{F}=F$ if and only if $F\left(\mathbf{x}_{\sigma}\right)=F(\mathbf{x})$ for all $\mathbf{x} \in[0,1]^{n}$ and $\sigma \in \mathcal{S}_{n}$.

c. $(\widehat{F})^{c}=\widehat{F}^{c}$.

For $(\widehat{F})^{c}(\mathbf{x})=1-\widehat{F}(\mathbf{x})=1-F\left(\mathbf{x}_{\sigma}\right)=F^{c}\left(\mathbf{x}_{\sigma}\right)=\widehat{F^{c}}(\mathbf{x})$ if $\mathbf{x} \in[0,1]^{n}$ and $\sigma \in \mathcal{S}_{n}$ is such that $\mathbf{x}_{\sigma} \in[0,1]_{(\geq)}^{n}$. 


\section{Linearity and ordered linearity}

In this section we study a relevant class of functions that are useful in order to introduce some examples and to describe some well-known functions (like the Łukasiewicz implication and the discrete Choquet integral).

Given $\mu \in \mathbb{R}$ and $\vec{v} \in \mathbb{R}^{n}$, let us consider the function $F: \mathbb{R}^{n} \rightarrow \mathbb{R}$ given by $F(\mathbf{x})=\mu+\mathbf{x} \cdot \vec{v}$ for all $\mathbf{x} \in \mathbb{R}^{n}$, where $\cdot$ denotes the usual scalar product, i.e., $\mathbf{x} \cdot \vec{v}=\sum_{i=1}^{n} x_{i} v_{i}$. The restriction of $F$ to $[0,1]^{n}$ is a function when $F\left([0,1]^{n}\right) \subseteq$ $[0,1]$.

Definition 4.1. We say that a pair $(\mu, \vec{v}) \in \mathbb{R} \times \mathbb{R}^{n}$ generates a linear fusion function if $\mu+\mathbf{x} \cdot \vec{v} \in[0,1]$ for all $\mathbf{x} \in[0,1]^{n}$. In such a case, we denote by $L[\mu, \vec{v}]$ the function

$$
L[\mu, \vec{v}](\mathbf{x})=\mu+\mathbf{x} \cdot \vec{v} \quad \text { for all } \mathbf{x} \in[0,1]^{n},
$$

which we call the $[\mu, \vec{v}]$-linear fusion function (or the fusion function generated by the pair $\left.(\mu, \vec{v}) \in \mathbb{R} \times \mathbb{R}^{n}\right)$.

Remark 4.2. We use the term fusion function to explicitly distinguish this class of functions from that of linear functions.

Lemma 4.3. Let $\vec{v} \in \mathbb{R}^{n}$ and consider the map $F:[0,1]^{n} \rightarrow \mathbb{R}$ given by $F(\mathbf{x})=$ $\mathbf{x} \cdot \vec{v}$. Set

$$
\mathcal{M}=\left\{\left(x_{1}, \ldots, x_{n}\right) \in[0,1]^{n} \mid x_{i} \in\{0,1\}, i=1, \ldots, n\right\} .
$$

Then, there exist $\mathbf{a}, \mathbf{b} \in \mathcal{M}$ such that $F(\mathbf{a})=\max F$ and $F(\mathbf{b})=\min F$.

Proof. Set $\mathcal{P}=\left\{\lambda_{i} \mid \lambda_{i}>0,1 \leq i \leq n\right\}, \mathcal{N}=\left\{\lambda_{i} \mid \lambda_{i}<0,1 \leq i \leq n\right\}$, where $\vec{v}=\left(\lambda_{1}, \ldots, \lambda_{n}\right)$. The claim follows from the obvious facts that $\max F=0$ if $\mathcal{P}=\emptyset, \max F=\sum_{\lambda \in \mathcal{P}} \lambda$ if $\mathcal{P} \neq \emptyset$ and $\min F=0$ if $\mathcal{N}=\emptyset$ and $\min F=\sum_{\lambda \in \mathcal{N}} \lambda$ if $\mathcal{N} \neq \emptyset$.

Proposition 4.4. The pair $[\mu, \vec{v}] \in \mathbb{R} \times \mathbb{R}^{n}$ defines a linear fusion function if and only if $0 \leq \mu+\sum_{i \in S} \lambda_{i} \leq 1$ for all $S \subseteq\{1, \ldots, n\}$, where $\vec{v}=\left(\lambda_{1}, \ldots, \lambda_{n}\right)$.

Proof. It follows immediately from Lemma 4.3

Corollary 4.5. If $[\mu, \vec{v}] \in \mathbb{R} \times \mathbb{R}^{n}$ satisfies the conditions in Proposition 4.4. then also $[1-\mu,-\vec{v}]$ satisfies them and $F=\mathrm{L}[\mu, \vec{v}]$ if and only if $F^{c}=\mathrm{L}[1-$ $\mu,-\vec{v}]$.

Proof. Note that $0 \leq \mu+\sum_{i \in S} \lambda_{i} \leq 1$ if and only if $1 \geq(1-\mu)+\sum_{i \in S}\left(-\lambda_{i}\right) \geq 0$, where $\vec{v}=\left(\lambda_{1}, \ldots, \lambda_{n}\right)$ and $S \subseteq\{1, \ldots, n\}$. 
Proposition 4.6. Assume that $[\mu, \vec{v}] \in \mathbb{R} \times \mathbb{R}^{n}$ defines the $[\mu, \vec{v}]$-linear fusion function $F:[0,1]^{n} \rightarrow[0,1]$. Then

(1) $\mathcal{D}^{\uparrow}(F)=\left\{\vec{r} \in \mathbb{R}^{n} \mid \vec{r} \cdot \vec{v} \geq 0\right\}$,

(2) $\mathcal{C}(F)=\left\{\vec{r} \in \mathbb{R}^{n} \mid \vec{r} \cdot \vec{v}=0\right\}$,

(3) $\mathcal{D}_{O D}^{\uparrow}(F)=\mathcal{D}_{S O D}^{\uparrow}(F)=\left\{\vec{r} \in \mathbb{R}^{n} \mid \vec{r}_{\sigma} \cdot \vec{v} \geq 0\right.$ for all $\left.\sigma \in \mathcal{S}_{n}\right\}$,

(4) $\mathcal{C}_{O D}(F)=\mathcal{C}_{S O D}(F)=\left\{\vec{r} \in \mathbb{R}^{n} \mid \vec{r}_{\sigma} \cdot \vec{v}=0\right.$ for all $\left.\sigma \in \mathcal{S}_{n}\right\}$.

Proof. (1) and (2) follow from the fact that $F(\mathbf{x}+c \vec{r})-F(\mathbf{x})=c \vec{r} \cdot \vec{v}$ and (3) and (4) from $F\left(\mathbf{x}+c \vec{r}_{\sigma^{-1}}\right)-F(\mathbf{x})=c \vec{r}_{\sigma^{-1}} \cdot \vec{v}$, if $\mathbf{x} \in[0,1]^{n}, c>0, \sigma \in \mathcal{S}_{n}$ and $\mathbf{x}_{\sigma}+c \vec{r} \in[0,1]^{n}$.

\section{Example 4.7.}

(1) The constant function $F:[0,1]^{n} \rightarrow[0,1]$, given by $F(\mathbf{x})=k$ for all $\mathbf{x} \in$ $[0,1]^{n}$, where $k \in[0,1]$, is the $[k, \mathbf{0}]$-linear fusion function.

(2) If $\mathbf{w}=\left(w_{1}, \ldots, w_{n}\right) \in[0,1]^{n}$ satisfies $\sum_{i=1}^{n} w_{i}=1$, the corresponding weighted average $F:[0,1]^{n} \rightarrow[0,1]$ given by $F(\mathbf{x})=\mathbf{x} \cdot \mathbf{w}$ if $\mathbf{x} \in[0,1]^{n}$ is the $[0, \mathbf{w}]$-linear fusion function.

Definition 4.8. We say that a pair $(\mu, \vec{v}) \in \mathbb{R} \times \mathbb{R}^{n}$ generates a ordered $(O)$ linear fusion function if $\mu+\mathbf{x}_{\sigma} \cdot \vec{v} \in[0,1]$ for all $\mathbf{x} \in[0,1]^{n}$ and $\sigma \in \mathcal{S}_{n}$ such that $\mathbf{x}_{\sigma} \in[0,1]_{(\geq)}^{n}$. In such a case, we denote by $O L[\mu, \vec{v}]$ the function

$$
O L[\mu, \vec{v}](\mathbf{x})=\mu+\mathbf{x}_{\sigma} \cdot \vec{v} \quad \text { for all } \mathbf{x} \in[0,1]^{n},
$$

which we call the ordered $[\mu, \vec{v}]$-linear fusion function.

Remark 4.9. Note that if $F=O L[\mu, \vec{v}]$, then $F\left(\mathbf{x}_{\sigma}\right)=F(\mathbf{x})$ for all $\sigma \in \mathcal{S}_{n}$. In particular, $F\left([0,1]^{n}\right)=F\left([0,1]_{(\geq)}^{n}\right)$.

Lemma 4.10. Let $\vec{v}=\left(\lambda_{1}, \ldots, \lambda_{n}\right) \in \mathbb{R}^{n}$ and consider the function $F$ : $[0,1]^{n} \rightarrow \mathbb{R}$ given by $F(\mathbf{x})=\mathbf{x}_{\sigma} \cdot \vec{v}$ if $\mathbf{x} \in[0,1]^{n}$, where $\sigma \in \mathcal{S}_{n}$ satisfies $\mathbf{x}_{\sigma} \in[0,1]_{(\geq)}^{n}$. Set

$$
\mathbf{u}_{0}=\mathbf{0}, \quad \mathbf{u}_{r}=(1, \ldots, \underset{r}{1}, 0, \ldots, 0) \in[0,1]^{n}, \text { for } r \in\{1, \ldots, n\} .
$$

Then there exist $j, k \in\{0,1, \ldots, n\}$ such that $\max _{\mathbf{x}} F(\mathbf{x})=F\left(\mathbf{u}_{j}\right)$ and $\min _{\mathbf{x}} F(\mathbf{x})=$ $F\left(\mathbf{u}_{k}\right)$.

Proof. Set $M=\max _{0 \leq i \leq n} F\left(\mathbf{u}_{i}\right)$ and $m=\min _{0 \leq i \leq n} F\left(\mathbf{u}_{i}\right)$. Proceed by induction on $n$. Let $n=1$. If $\lambda_{1} \geq 0$, then $M=F(1)=\max _{x} F(x)$ and $m=F(0)=\min _{x} F(x)$. If $\lambda_{1} \leq 0$, then $M=F(0)=\max _{x} F(x)$ and $m=F(1)=\min _{x} F(x)$.

Let now $n>1$ and consider the case of the maximum. Let us suppose that there exists $\mathbf{x} \in[0,1]^{n}$ such that $M<F(\mathbf{x})$. Since $F\left(\mathbf{u}_{0}\right)=0$, we have that 
$F(\mathbf{x})>0$. We may assume, without loss of generality, that $x_{1} \geq \ldots \geq x_{n}$, where $=\left(x_{1}, \ldots, x_{n}\right)$. As $F(\mathbf{x})>0$, then $x_{1}>0$, and

$$
\begin{aligned}
\mathbf{x} \cdot \vec{v} & =x_{1} \lambda_{1}+\ldots+x_{n} \lambda_{n} \\
& =x_{1}\left(\lambda_{1}+\frac{x_{2}}{x_{1}} \lambda_{2}+\ldots+\frac{x_{n}}{x_{1}} \lambda_{n}\right) \\
& \leq \lambda_{1}+\frac{x_{2}}{x_{1}} \lambda_{2}+\ldots+\frac{x_{n}}{x_{1}} \lambda_{n} \\
& =\frac{1}{x_{1}} \mathbf{x} \cdot \vec{v}
\end{aligned}
$$

315 as $\lambda_{1}+\frac{x_{2}}{x_{1}} \lambda_{2}+\ldots+\frac{x_{n}}{x_{1}} \lambda_{n}>0$ and $x_{1} \in[0,1]$. Set now $\mathbf{y}=\left(y_{1}, \ldots, y_{n}\right)=\frac{1}{x_{1}} \mathbf{x}$, and observe that $1=y_{1} \geq \ldots \geq y_{n}$.

If we consider $\mathbf{z}=\left(z_{2}, \ldots, z_{n}\right) \in[0,1]^{n-1}$, then by the induction hypothesis, the scalar product $\left(z_{2}, \ldots, z_{n}\right) \cdot\left(\lambda_{2}, \ldots, \lambda_{n}\right)$ reaches an absolute maximum for some

$$
\mathbf{t}=\left(t_{2}, \ldots, t_{n}\right) \in\left\{(1, \ldots, \underset{r}{1}, 0, \ldots, 0) \in[0,1]^{n-1} \mid 0 \leq r \leq n-1\right\} .
$$

Thus,

$$
\begin{aligned}
M & <F(\mathbf{x})=\lambda_{1}+\left(y_{2}, \ldots, y_{n}\right) \cdot\left(\lambda_{2}, \ldots, \lambda_{n}\right) \\
& \leq \lambda_{1}+\mathbf{t} \cdot\left(\lambda_{2}, \ldots, \lambda_{n}\right)=\left(1, t_{2}, \ldots, t_{n}\right) \cdot\left(\lambda_{1}, \ldots, \lambda_{n}\right),
\end{aligned}
$$

where $\left(1, t_{2}, \ldots, t_{n}\right) \in\left\{\mathbf{u}_{0}, \mathbf{u}_{1}, \ldots, \mathbf{u}_{n}\right\}$, which contradicts the election of $M$. Observe that $(-F)(\mathbf{x})=\mathbf{x}_{\sigma} \cdot(-\vec{v})$. So $\min F=\max (-F)$.

Remark 4.11. In the conditions of Lemma 4.10, as $F$ is a continuous function on a compact set of $\mathbb{R}^{n}$, we know of the existence of a maximum and a minimum, but this fact is not used in the proof of Lemma 4.10. (A similar remark can be made about Proposition 4.4.).

Proposition 4.12. The pair $[\mu, \vec{v}] \in \mathbb{R} \times \mathbb{R}^{n}$, where $\vec{v}=\left(\lambda_{1}, \ldots, \lambda_{n}\right)$, defines an $O$ linear fusion function if and only if $0 \leq \mu \leq 1$ and $0 \leq \mu+\sum_{i=1}^{r} \lambda_{i} \leq 1$ 325 for all $r \in\{1, \ldots, n\}$.

Proof. It is an immediate consequence of Lemma 4.10 .

Corollary 4.13. If $F:[0,1]^{n} \rightarrow[0,1]$ is the $[\mu, \vec{v}]$-linear fusion function, then $\widehat{F}$ is the $O[\mu, \vec{v}]$-linear fusion function.

Analogously as for Corollary 4.5 we have the following result.

330 Corollary 4.14. Let $[\mu, \vec{v}] \in \mathbb{R} \times \mathbb{R}^{n}$ satisfying the conditions of Corollary 4.12. Then, the pair $[1-\mu,-\vec{v}]$ also satisfies the conditions of Corollary 4.12 and $F=\mathrm{OL}[\mu, \vec{v}]$ if and only if $F^{c}=\mathrm{OL}[1-\mu,-\vec{v}]$.

Proposition 4.15. Let $[\mu, \vec{v}] \in \mathbb{R} \times \mathbb{R}^{n}$ satisfying the conditions of Corollary 4.12 and $F=\mathrm{OL}[\mu, \vec{v}]$. Then 
(1) $\mathcal{D}_{O D}^{\uparrow}(F)=\left\{\vec{r} \in \mathbb{R}^{n} \mid \vec{r} \cdot \vec{v} \geq 0\right\}$

(2) $\mathcal{C}_{O D}(F)=\left\{\vec{r} \in \mathbb{R}^{n} \mid \vec{r} \cdot \vec{v}=0\right\}$.

Proof. Let $\mathbf{x} \in[0,1]^{n}, c>0$ and $\sigma \in \mathcal{S}_{n}$ such that $\mathbf{x}_{\sigma}, \mathbf{x}_{\sigma}+c \vec{r} \in[0,1]_{(\geq)}^{n}$. Then $F\left(\mathbf{x}+c \vec{r}_{\sigma^{-1}}\right)=\mu+\left(\mathbf{x}_{\sigma}+c \vec{r}\right) \cdot \vec{v}=F(\mathbf{x})+c \vec{r} \cdot \vec{v}$ and the thesis follows.

In several occasions we focus on the particular case $n=2$. Observe that if $\sigma \in \mathcal{S}_{2}$, we have that $\sigma^{-1}=\sigma$.

The following auxiliary result follows immediately from a simple geometric approach.

Lemma 4.16. Let $\vec{r}=\left(r_{1}, r_{2}\right) \in \mathbb{R}^{2}$.

(1) There exist $\mathbf{y} \in[0,1]_{(\geq)}^{2}$ and $c>0$ such that $\mathbf{y}+c \vec{r} \in[0,1]_{(\geq)}^{2}$.

${ }_{345}$ (2) There exist $\mathbf{y} \in[0,1]_{(\leq)}^{2}$ and $c>0$ such that $\mathbf{y}+c \vec{r} \in[0,1]_{(\leq)}^{2}$.

(3) If $\vec{r} \in \mathbb{R}_{(>)}^{2}$ there exist $\mathbf{y} \in[0,1]_{(<)}^{2}$ and $c>c^{\prime}>0$ such that $\mathbf{y}+c \vec{r} \in[0,1]_{(>)}^{2}$ and $\mathbf{y}+c^{\prime} \vec{r} \in[0,1]_{(=)}^{2}$.

(4) If $\vec{r} \in \mathbb{R}_{(<)}^{2}$ there exist $\mathbf{y} \in[0,1]_{(>)}^{2}$ and $c>c^{\prime}>0$ such that $\mathbf{y}+c \vec{r} \in[0,1]_{(<)}^{2}$ and $\mathbf{y}+c^{\prime} \vec{r} \in[0,1]_{(=)}^{2}$.

350 Proposition 4.17. Let $[\mu, \vec{v}] \in \mathbb{R} \times \mathbb{R}^{2}$ satisfying the conditions of Corollary 4.12 and $F=\mathrm{OL}[\mu, \vec{v}]$. Then

(1) $\mathcal{D}_{O D}^{\uparrow}(F)=\left\{\vec{r} \in \mathbb{R}^{2} \mid \vec{r} \cdot \vec{v} \geq 0\right\}$.

(2) $\mathcal{C}_{O D}(F)=\left\{\vec{r} \in \mathbb{R}^{2} \mid \vec{r} \cdot \vec{v}=0\right\}$.

(3) $\mathcal{D}_{S O D}^{\uparrow}(F)=\left\{\vec{r} \in \mathbb{R}_{(\geq)}^{2} \mid \vec{r} \cdot \vec{v} \geq 0\right\} \cup\left\{\vec{r} \in \mathbb{R}_{(\leq)}^{2} \mid \vec{r} \cdot \vec{v} \geq 0\right.$ and $\left.\vec{r} \cdot \vec{v}^{d} \geq 0\right\}$.

(4) $\mathcal{C}_{S O D}(F)=\left\{\vec{r} \in \mathbb{R}_{(\geq)}^{2} \mid \vec{r} \cdot \vec{v}=0\right\} \cup\left\{\vec{r} \in \mathbb{R}_{(\leq)}^{2} \mid \vec{r} \cdot \vec{v}=\vec{r} \cdot \vec{v} d=0\right\}$.

(5) $\mathcal{D}^{\uparrow}(F)=\left\{\vec{r} \in \mathbb{R}^{2} \mid \vec{r} \cdot \vec{v} \geq 0\right.$ and $\left.\vec{r} \cdot \vec{v}^{d} \geq 0\right\}$.

(6) $\mathcal{C}(F)=\left\{\vec{r} \in \mathbb{R}^{2} \mid \vec{r} \cdot \vec{v}=\vec{r} \cdot \vec{v}^{d}=0\right\}$.

Proof. (1) and (2) are particular cases of Proposition 4.15 .

Let $\mathbf{x} \in[0,1]^{2}, c>0$ and $\sigma \in \mathcal{S}_{2}$ such that $\mathbf{x}_{\sigma} \in[0,1]_{(\geq)}^{2}$ and $\mathbf{x}_{\sigma}+c \vec{r} \in[0,1]^{2}$. If actually $\mathbf{x}_{\sigma}+c \vec{r} \in[0,1]_{(\geq)}^{2}$, then we have

$$
F\left(\mathbf{x}+c \vec{r}_{\sigma}\right)-F(\mathbf{x})=c \vec{r} \cdot \vec{v} .
$$


Let us assume that $x_{\sigma(1)}+c r_{1}<x_{\sigma(2)}+c r_{2}$ (then necessarily is $r_{1}<r_{2}$ ). Then $F\left(\mathbf{x}+c \vec{r}_{\sigma}\right)=\mu+\left(x_{\sigma(2)}+c r_{2}\right) \lambda_{1}+\left(x_{\sigma(1)}+c r_{1}\right) \lambda_{2}$. Thus

$$
F\left(\mathbf{x}+c \vec{r}_{\sigma}\right)-F(\mathbf{x})=\left(x_{\sigma(1)}-x_{\sigma(2)}\right)\left(\lambda_{2}-\lambda_{1}\right)+c \vec{r} \cdot \vec{v}^{d} .
$$

Observe that

$$
\vec{r} \cdot \vec{v}^{d}=\vec{r} \cdot \vec{v}+\left(r_{2}-r_{1}\right)\left(\lambda_{1}-\lambda_{2}\right) .
$$

(3) Let us assume that $F$ is SOD $\vec{r}$-increasing. If $r_{1} \geq r_{2}$, then we are in (A). By Lemma 4.16, if $r_{1}<r_{2}$ both situations (A) and (B), in the last case also with $x_{\sigma(1)}=x_{\sigma(2)}$, can occur. Therefore

$$
\mathcal{D}_{\mathrm{SOD}}^{\uparrow}(F) \subseteq\left\{\vec{r} \in \mathbb{R}_{(\geq)}^{2} \mid \vec{r} \cdot \vec{v} \geq 0\right\} \cup\left\{\vec{r} \in \mathbb{R}_{(\leq)}^{2} \mid \vec{r} \cdot \vec{v} \geq 0 \text { and } \vec{r} \cdot \vec{v}^{d} \geq 0\right\} .
$$

Conversely, if $r_{1} \geq r_{2}$ and $\vec{r} \cdot \vec{v} \geq 0$ then (see A) $F\left(\mathbf{x}+c \vec{r}_{\sigma}\right) \geq F(\mathbf{x})$.

Let $r_{1}<r_{2}, \vec{r} \cdot \vec{v} \geq 0, \vec{r} \cdot \vec{v}^{d} \geq 0$.

If $\lambda_{1} \leq \lambda_{2}$, then (see (A) and (B)) $F\left(\mathbf{x}+c \vec{r}_{\sigma}\right) \geq F(\mathbf{x})$.

Suppose that $\lambda_{1}>\lambda_{2}$. If $x_{\sigma(1)}=x_{\sigma(2)}$, then $F\left(\mathbf{x}+c \vec{r}_{\sigma}\right) \geq F(\mathbf{x})$. Suppose further that $x_{\sigma(1)}>x_{\sigma(2)}$. With $x_{\sigma(1)}-x_{\sigma(2)}=x, c\left(r_{2}-r_{1}\right)=r$, we have $0<x<r$. So $c \vec{r} \cdot \vec{v}^{d}=c \vec{r} \cdot \vec{v}+r\left(\lambda_{1}-\lambda_{2}\right)$ (see (C)) and

$$
\begin{aligned}
F\left(\mathbf{x}+c \vec{r}_{\sigma}\right)-F(\mathbf{x}) & =x\left(\lambda_{2}-\lambda_{1}\right)+c \vec{r} \cdot \vec{v}+r\left(\lambda_{1}-\lambda_{2}\right) \\
& =(r-x)\left(\lambda_{1}-\lambda_{2}\right)+c \vec{r} \cdot \vec{v}>0,
\end{aligned}
$$

hence $F$ is SOD $\vec{r}$-increasing.

(4) Arguing as in (3) we deduce that

$$
\mathcal{C}_{\mathrm{SOD}}(F) \subseteq\left\{\vec{r} \in \mathbb{R}_{(\geq)}^{2} \mid \vec{r} \cdot \vec{v}=0\right\} \cup\left\{\vec{r} \in \mathbb{R}_{(\leq)}^{2} \mid \vec{r} \cdot \vec{v}=\vec{r} \cdot \vec{v}^{d}=0\right\} .
$$

If $r_{1} \geq r_{2}$ and $\vec{r} \cdot \vec{v}=0$, then $F\left(\mathbf{x}+c \vec{r}_{\sigma}\right)=F(\mathbf{x})$ (see (A)). Assume now that $r_{1}<r_{2}, \vec{r} \cdot \vec{v}=\vec{r} \cdot \vec{v}^{d}=0$. We have so $\left(r_{2}-r_{1}\right)\left(\lambda_{1}-\lambda_{2}\right)=0$ (see (C)) and therefore it must be $\lambda_{1}=\lambda_{2}$ and so $F\left(\mathbf{x}+c \vec{r}_{\sigma}\right)=F(\mathbf{x})$ (see (B)) also in this case.

Let now $\mathbf{x} \in[0,1]^{2}$ and $c>0$ such that $\mathbf{x}+c \vec{r} \in[0,1]^{2}$. We have the following possibilities (set $d=F(\mathbf{x}+c \vec{r})-F(\mathbf{x}))$ :

- $\mathbf{x} \in[0,1]_{(\geq)}^{2}$ and

(a) $\mathbf{x}+c \vec{r} \in[0,1]_{(\geq)}^{2}$, when $d=c \vec{r} \cdot v$, or

(b) $x_{1}+c r_{1} \leq x_{2}+c r_{2}$, when $d=\left(x_{1}-x_{2}\right)\left(\lambda_{2}-\lambda_{1}\right)+c \vec{r} \cdot \vec{v}^{d}$ (and necessarily $r_{1} \leq r_{2}$ ), or

- $\mathbf{x} \in[0,1]_{(\leq)}^{2}$ and

(c) $x_{1}+c r_{1} \geq x_{2}+c r_{2}$, when $d=\left(x_{2}-x_{1}\right)\left(\lambda_{2}-\lambda_{1}\right)+c \vec{r} \cdot \vec{v}$ (and necessarily $r_{1} \geq r_{2}$ ), or

(d) $\mathbf{x}+c \vec{r} \in[0,1]_{(\leq)}^{2}$, when $d=c \vec{r} \cdot \vec{v}^{d}$. 
(5) Therefore if $F$ is $\vec{r}$-increasing, necessarily $\vec{r} \cdot \vec{v} \geq 0$ and $\vec{r} \cdot \vec{v}^{d} \geq 0$. Let us assume that this happens. If we are in the case $(a)$ or $(d)$, then $d \geq 0$. If we are in the case $(b)$ and $\lambda_{2} \geq \lambda_{1}$, then $d \geq 0$. If $\lambda_{2}<\lambda_{1}$ then (see (C))

$$
d=\left[c\left(r_{2}-r_{1}\right)-\left(x_{1}-x_{2}\right)\right]\left(\lambda_{1}-\lambda_{2}\right)+c \vec{r} \cdot \vec{v} \geq 0
$$

since $c\left(r_{2}-r_{1}\right) \geq x_{1}-x_{2}$ because $x_{2}+c r_{2} \geq x_{1}+c r_{1}$. Proceed analogously in the case $(c)$.

(6) Proceed analogously as in (4).

Remark 4.18. Let $\left[\mu,\left(\lambda_{1}, \lambda_{2}\right)\right] \in \mathbb{R} \times \mathbb{R}^{2}$ satisfying the conditions of Corollary 4.12 and let $F$ the corresponding $\mathrm{O}$ linear fusion function. We may simplify some expressions of Proposition 4.17 in some cases. From (C) in Proposition 4.17 we deduce that if $\lambda_{1} \geq \lambda_{2}$ then $r_{1} \leq r_{2}$ implies $\vec{r} \cdot \vec{v} d \geq \vec{r} \cdot \vec{v}$. So if $\lambda_{1} \geq \lambda_{2}$, then

$$
\mathcal{D}_{\mathrm{SOD}}^{\uparrow}(F)=\mathcal{D}_{\mathrm{OD}}^{\uparrow}(F)=\left\{\vec{r} \in \mathbb{R}^{2} \mid \vec{r} \cdot \vec{v} \geq 0\right\} .
$$

Analogously, if $\vec{r} \cdot \vec{v}=0$, then $\vec{r} \cdot \vec{v}^{d}=\left(r_{2}-r_{1}\right)\left(\lambda_{1}-\lambda_{2}\right)$. So, in this case, $\vec{r} \cdot \vec{v}^{d}=0$ if and only if either $r_{1}=r_{2}$ or $\lambda_{1}=\lambda_{2}$. Therefore

- If $\lambda_{1} \neq \lambda_{2}$ then $\mathcal{C}_{\mathrm{SOD}}(F)=\mathcal{C}(F)=\left\{\vec{r} \in \mathbb{R}_{(\geq)}^{2} \mid \vec{r} \cdot \vec{v}=0\right\}$.

- If $\lambda_{1}=\lambda_{2}$ then $\mathcal{C}_{\mathrm{SOD}}(F)=\mathcal{C}_{\mathrm{OD}}(F)=\mathcal{C}(F)=\left\{\vec{r} \in \mathbb{R}^{2} \mid \vec{r} \cdot \vec{v}=0\right\}$.

Example 4.19. Let us present some examples of $O$ linear fusion functions. We omit the mentions $\mathbf{x} \in[0,1]^{n}$ and $\sigma \in \mathcal{S}_{n}$ is such that $\mathbf{x}_{\sigma} \in[0,1]_{(\geq)}^{n}$.

(1) If $\mathbf{a}=\left(a_{1}, \ldots, a_{n}\right) \in[0,1]_{(\geq)}^{n}$, put $\widetilde{\mathbf{a}}=\left(a_{1},-a_{2}, \ldots,(-1)^{n-1} a_{n}\right)$. Then we have the $\mathrm{O}[0, \widetilde{\mathbf{a}}]$-linear fusion function $F_{\mathbf{a}}:[0,1]^{n} \rightarrow[0,1]$ given by

$$
F_{\mathbf{a}}(\mathbf{x})=x_{\sigma(1)} a_{1}-x_{\sigma(2)} a_{2}+\cdots+(-1)^{n-1} x_{\sigma(n)} a_{n}
$$

(2) If $\lambda \in[0,1]$, the $\mathrm{O}[0,(1,-\lambda)]$-linear fusion function $F:[0,1]^{2} \rightarrow[0,1]$ given by

$$
F(\mathbf{x})=\max \left(x_{1}, x_{2}\right)-\lambda \min \left(x_{1}, x_{2}\right) .
$$

Observe that $F^{c}:[0,1]^{2} \rightarrow[0,1]$ is the $\mathrm{O}[1,(-1, \lambda)]$-linear fusion function given by

$$
F^{c}(\mathbf{x})=1-\max \left(x_{1}, x_{2}\right)+\lambda \min \left(x_{1}, x_{2}\right) .
$$

(3) The $\mathrm{O}[0,(1,-1)]$-linear fusion function $F:[0,1]^{2} \rightarrow[0,1]$ given by

$$
F(\mathbf{x})=\left|x_{1}-x_{2}\right|
$$

Then $F^{c}:[0,1]^{2} \rightarrow[0,1]$ is the $\mathrm{O}[1,(-1,1)]$-linear fusion function given by

$$
F^{c}(\mathbf{x})=1-\left|x_{1}-x_{2}\right|,
$$

which is a restricted equivalence function (see [4]). 
(4) The $\mathrm{O}\left[\frac{1}{2},\left(\frac{1}{2},-1\right)\right]$-linear fusion function $F:[0,1]^{2} \rightarrow[0,1]$ given by

$$
F(\mathbf{x})=\frac{1}{2}\left(1+\max \left(x_{1}-x_{2}\right)-2 \min \left(x_{1}-x_{2}\right)\right) .
$$

(5) The $\mathrm{O}\left[1,\left(-1, \frac{1}{2}\right)\right]$-linear fusion function $F:[0,1]^{2} \rightarrow[0,1]$ given by

$$
F(\mathbf{x})=1-\max \left(x_{1}, x_{2}\right)+\frac{1}{2} \min \left(x_{1}, x_{2}\right) .
$$

(6) Let $\mathbf{w} \in[0,1]^{n}$ with $\sum_{i=1}^{n} w_{i}=1$, the OWA operator $A:[0,1]^{n} \rightarrow[0,1]$ with respect to the weighting vector $\mathbf{w}$, given by

$$
A(\mathbf{x})=\mathbf{x}_{\sigma} \cdot \mathbf{w}
$$

is the $\mathrm{O}[0, \mathbf{w}]$-linear fusion function. Observe that if $F:[0,1]^{n} \rightarrow[0,1]$ is the weighted average corresponding to $\mathbf{w}$, then $A=\widehat{F}$ (see Example 4.7 (2) and Corollary 4.13.

Example 4.20. Let $p>0$ and $\vec{r}=(t, \ldots, t, s) \in \mathbb{R}^{n}$, where $s \leq t$. The function $F:[0,1]^{n} \rightarrow[0,1]$ given by

$$
F(\mathbf{x})=\frac{1}{n-1} \sum_{j=2}^{n}\left|x_{1}-x_{j}\right|^{p},
$$

if $\mathbf{x}=\left(x_{1}, \ldots, x_{n}\right) \in[0,1]^{n}$, is SOD $\vec{r}$-increasing.

Indeed, given $\mathbf{x} \in[0,1]^{n}, \sigma \in \mathcal{S}_{n}$ and $c \in \mathbb{R}^{+}$such that $\mathbf{x}_{\sigma} \in[0,1]_{(\geq)}^{n}$, $\mathbf{x}_{\sigma}+c \vec{r} \in[0,1]^{n}$. Observe that

$$
\mathbf{x}+c \vec{r}_{\sigma^{-1}}=\left(x_{1}+c t, \ldots, x_{i-1}+c t, x_{i}+c s, x_{i+1}+c t, \ldots, x_{n}+c t\right),
$$

where $i=\sigma(n)$ and that $x_{\sigma(n)}=\min \left\{x_{1}, \ldots, x_{n}\right\}$, so that $x_{1} \geq x_{i}$. As $t \geq s$, we have

$$
\begin{aligned}
F\left(\mathbf{x}+c \vec{r}_{\sigma^{-1}}\right) & =\frac{1}{n-1}\left(\sum_{\substack{j=2 \\
j \neq i}}^{n}\left|x_{1}-x_{j}\right|^{p}+\left|\left(x_{1}-x_{i}\right)+c(t-s)\right|^{p}\right) \\
& \geq \frac{1}{n-1} \sum_{j=2}^{n}\left|x_{1}-x_{j}\right|^{p}=F(\mathbf{x}),
\end{aligned}
$$

as required. Note that, in the case where $\sigma(n)=1$, the result follows readily since $x_{1} \leq x_{j}$ and $s \leq t$. Thus,

$$
\left|x_{1}-x_{j}+c s-c t\right| \geq\left|x_{1}-x_{j}\right| .
$$


Corollary 4.21. Let $0<p \in \mathbb{R}, \vec{r}=\left(r_{1}, r_{2}\right) \in \mathbb{R}^{2}$ and consider the function $F:[0,1]^{2} \rightarrow[0,1]$ given by

$$
F\left(x_{1}, x_{2}\right)=\left|x_{1}-x_{2}\right|^{p}
$$

if $\left(x_{1}, x_{2}\right) \in[0,1]^{2}$. Then

(1) $\mathcal{D}_{S O D}^{\uparrow}(F)=\mathcal{D}_{O D}^{\uparrow}(F)=\left\{\vec{r} \in \mathbb{R}^{2} \mid r_{1} \geq r_{2}\right\}$.

(2) $\mathcal{D}^{\uparrow}(F)=\mathcal{C}(F)=\mathcal{C}_{S O D}(F)=\mathcal{C}_{O D}(F)=\left\{\vec{r} \in \mathbb{R}^{2} \mid r_{1}=r_{2}\right\}$.

Proof. Consider $\varphi:[0,1] \rightarrow[0,1]$ given by $\varphi(x)=x^{p}$ if $x \in[0,1]$. Then $F=$ $\varphi \circ F_{1}$, where $F_{1}(\mathbf{x})=\left|x_{1}-x_{2}\right|$, so it is consequence of Propositions 3.8 and 4.17.

Proposition 4.22. Let $0<p \in \mathbb{R}$ and consider the function $F:[0,1]^{2} \rightarrow[0,1]$ given by

$$
F(\mathbf{x})=1-\left|x_{1}-x_{2}\right|^{p}
$$

if $\mathbf{x}=\left(x_{1}, x_{2}\right) \in[0,1]^{2}$. Set $\vec{r}=\left(r_{1}, r_{2}\right)$. Then

(1) $\mathcal{D}^{\uparrow}(F)=\mathcal{D}_{S O D}^{\uparrow}(F)=\mathcal{C}(F)=\mathcal{C}_{S O D}(F)=\mathcal{C}_{O D}(F)=$

$$
=\left\{\vec{r} \in \mathbb{R}^{2} \mid r_{1}=r_{2}\right\} \text {. }
$$

(2) $\mathcal{D}_{O D}^{\uparrow}(F)=\left\{\vec{r} \in \mathbb{R}^{2} \mid r_{1} \leq r_{2}\right\}$.

Proof. Let $M:[0,1]^{2} \rightarrow[0,1]$ given by $M(\mathbf{x})=\left|x_{1}-x_{2}\right|^{p}$ if $\mathbf{x}=\left(x_{1}, x_{2}\right) \in[0,1]^{2}$. Then $F=M^{c}$, hence, by Proposition 3.5 and Corollary 4.21, it only rests to show the assertion on $\mathcal{D}_{\text {SOD }}^{\uparrow}(F)$. By Lemma 3.9. $\left\{\vec{r} \in \mathbb{R}^{2} \mid r_{1}=r_{2}\right\}=\mathcal{D}^{\uparrow}(F) \subseteq$ $\mathcal{D}_{\text {SOD }}^{\uparrow}(F)$. Assume that $F$ is SOD $\vec{r}$-increasing. Take $0<x<1$ and $\mathbf{x}=(x, x)$, 405 so $\mathbf{x} \in[0,1]_{(\geq)}^{2}$; take $c>0$ such that $\mathbf{x}+c \vec{r} \in[0,1]^{2}$. Then $F(\mathbf{x}+c \vec{r})=$ $1-\left|c\left(r_{1}-r_{2}\right)\right|^{p}$. As $F$ is SOD $\vec{r}$-increasing, it must be $F(\mathbf{x}+c \vec{r}) \geq F(\mathbf{x})=1$, hence $r_{1}=r_{2}$.

\section{An operation between functions}

We introduce here an operation between functions from $[0,1]^{2}$ to $[0,1]$ which generalizes, when applied to O linear fusion functions, for $n=2$, the Choquet integral and the Łukasiewicz implication.

Definition 5.1. Let $F_{i}:[0,1]^{2} \rightarrow[0,1], i=1,2$, be two functions such that $F_{1}(x, x)=F_{2}(x, x)$ for all $x \in[0,1]$. Define $F_{1} * F_{2}:[0,1]^{2} \rightarrow[0,1]$ by

$$
\left(F_{1} * F_{2}\right)(\mathbf{x})=\left\{\begin{array}{l}
F_{1}(\mathbf{x}) \text { if } \mathbf{x} \in[0,1]_{(\geq)}^{2}, \\
F_{2}(\mathbf{x}) \text { if } \mathbf{x} \in[0,1]_{(\leq)}^{2} .
\end{array}\right.
$$


Proposition 5.2. Let $F_{i}:[0,1]^{2} \rightarrow[0,1], i=1,2$, be two functions such that $F_{1}(x, x)=F_{2}(x, x)$ for all $x \in[0,1]$. Then, for $T \in\{\emptyset, S O D, O D\}$, the following hold.

(1) $\mathcal{D}_{T}^{\uparrow}\left(F_{1}\right) \cap \mathcal{D}_{T}^{\uparrow}\left(F_{2}\right) \subseteq \mathcal{D}_{T}^{\uparrow}\left(F_{1} * F_{2}\right)$.

(2) $\mathcal{C}_{T}\left(F_{1}\right) \cap \mathcal{C}_{T}\left(F_{2}\right) \subseteq \mathcal{C}_{T}\left(F_{1} * F_{2}\right)$

Proof. Set in this proof $F=F_{1} * F_{2}$.

- (1) and (2) for $\mathrm{T}=\mathrm{OD}$.

Let $\mathbf{x} \in[0,1]^{2}, \sigma \in \mathcal{S}_{2}, c>0$ such that $\mathbf{x}_{\sigma}, \mathbf{x}_{\sigma}+c \vec{r} \in[0,1]_{(\geq)}^{2}$. These assertions follow immediately taking the following into account.

(a) If $\sigma=\mathrm{id}, F(\mathbf{x}+c \vec{r})=F_{1}(\mathbf{x}+c \vec{r}), F(\mathbf{x})=F_{1}(\mathbf{x})$.

(b) If $\sigma=(12), F\left(\mathbf{x}+c \vec{r}_{\sigma}\right)=F_{2}\left(\mathbf{x}+c \vec{r}_{\sigma}\right), F(\mathbf{x})=F_{2}(\mathbf{x})$.

- (1) and (2) for $\mathrm{T}=\emptyset$.

Let $\mathbf{x} \in[0,1]^{2}$ and $c>0$ such that $\mathbf{x}+c \vec{r} \in[0,1]^{2}$.

Assume that $\vec{r} \in \mathcal{D}^{\uparrow}\left(F_{1}\right) \cap \mathcal{D}^{\uparrow}\left(F_{2}\right)$. If $\mathbf{x}, \mathbf{x}+c \vec{r} \in[0,1]_{(\geq)}^{2}$, then $F(\mathbf{x})=$ $F_{1}(\mathbf{x}) \leq F_{1}(\mathbf{x}+c \vec{r})=F(\mathbf{x}+c \vec{r})$ and $\vec{r} \in \mathcal{D}^{\uparrow}(F)$. Analogously if $\mathbf{x}, \mathbf{x}+c \vec{r} \in$ $[0,1]_{(\leq)}^{2}$. Suppose $\mathbf{x} \in[0,1]_{(\geq)}^{2}, \mathbf{x}+c \vec{r} \in[0,1]_{(<)}^{2}$. Then $r_{2}>r_{1}$. By Lemma 4.16 there exists $c^{\prime}>0, c>c^{\prime}$, such that, with $\mathbf{z}=\left(z_{1}, z_{2}\right)=\mathbf{x}+\left(c-c^{\prime}\right) \vec{r}$, we have $z_{1}=z_{2}$; as $\mathbf{z}+c^{\prime} \vec{r}=\mathbf{x}+c \vec{r}$, one has, with $c^{\prime \prime}=c-c^{\prime}$,

$F(\mathbf{x})=F_{1}(\mathbf{x}) \leq F_{1}\left(\mathbf{x}+c^{\prime \prime} \vec{r}\right)=F_{1}(\mathbf{z})=F_{2}(\mathbf{z}) \leq F_{2}\left(\mathbf{z}+c^{\prime} \vec{r}\right)=F(\mathbf{x}+c \vec{r})$.

Analogously for (2).

- (1) and (2) for $\mathrm{T}=\mathrm{SOD}$.

Assume that $\vec{r} \in \mathcal{D}_{\mathrm{SOD}}^{\uparrow}\left(F_{1}\right) \cap \mathcal{D}_{\mathrm{SOD}}^{\uparrow}\left(F_{2}\right)$. Let $\mathbf{x} \in[0,1]^{2}, \sigma \in \mathcal{S}_{2}$ and $c>0$ such that $\mathbf{x}_{\sigma} \in[0,1]_{(\geq)}^{2}, \mathbf{x}_{\sigma}+c \vec{r} \in[0,1]^{2}$.

(a) Suppose that $\sigma=\mathrm{id}$, so $\mathbf{x} \in[0,1]_{(\geq)}^{2}$.

- If $\mathbf{x}+c \vec{r} \in[0,1]_{(\geq)}^{2}$, then $F(\mathbf{x}+c \vec{r})=F_{1}(\mathbf{x}+c \vec{r}) \geq F_{1}(\mathbf{x})=F(\mathbf{x})$.

- If $\mathbf{x}+c \vec{r} \in[0,1]_{(<)}^{2}$, then $r_{1}<r_{2}$ and we may proceed as in the case $\mathrm{T}=\emptyset$

(b) Suppose that $\sigma=(12)$, so $\mathbf{x} \in[0,1]_{(\leq)}^{2}$.

- If $\mathbf{x}+c \vec{r}_{\sigma} \in[0,1]_{(\leq)}^{2}, F\left(\mathbf{x}+c \vec{r}_{\sigma}\right)=F_{2}\left(\mathbf{x}+c \vec{r}_{\sigma}\right) \geq F_{2}(\mathbf{x})=F(\mathbf{x})$.

- Assume that $\mathbf{x}+c \vec{r}_{\sigma} \in[0,1]_{(>)}^{2}$. Apply Lemma 4.16 to $\vec{s}=\vec{r}_{\sigma}$ and argue analogous to the case $\mathrm{T}=\emptyset$.

Analogously for (2). 
Lemma 5.3. Let $G=O L[\mu, \vec{a}]$ and $H=O L[\nu, \vec{b}]$, where $[\mu, \vec{a}],[\nu, \vec{b}]$ belong to $\mathbb{R} \times \mathbb{R}^{2}$ and satisfy the conditions of Corollary 4.12, and set $\vec{a}=\left(a_{1}, a_{2}\right)$, $\vec{b}=\left(b_{1}, b_{2}\right)$. We have that $G(x, x)=H(x, x)$ for all $x \in[0,1]$ if and only if $\mu=\nu$ and $a_{1}+a_{2}=b_{1}+b_{2}$.

Proof. $(\Rightarrow)$ With $x=0$ we have $\mu=\nu$. Take now for instance $x=1$ and obtain $a_{1}+a_{2}=b_{1}+b_{2}$.

$(\Leftarrow)$ Follows immediately.

Proposition 5.4. Let $\vec{a}=\left(a_{1}, a_{2}\right), \vec{b}=\left(b_{1}, b_{2}\right) \in \mathbb{R}^{2}$ be such that $s=a_{1}+a_{2}=$ $b_{1}+b_{2}$ and suppose that $[\mu, \vec{a}],[\mu, \vec{b}] \in \mathbb{R} \times \mathbb{R}^{2}$ satisfy the conditions of Proposition 3.7. Let $G=O L[\mu, \vec{a}]$ and $H=O L[\mu, \vec{b}]$ and consider $G * H$ (recall Lemma 4.3). Then the following statements hold.

(1) $\mathcal{D}_{O D}^{\uparrow}(G * H)=\left\{\vec{r} \in \mathbb{R}^{2} \mid \vec{r} \cdot \vec{a} \geq 0, \vec{r} \cdot \vec{b} \geq 0\right\}$.

(2) $\mathcal{C}_{O D}(G * H)=\left\{\vec{r} \in \mathbb{R}^{2} \mid \vec{r} \cdot \vec{a}=\vec{r} \cdot \vec{b}=0\right\}$.

(3) $\mathcal{D}_{S O D}^{\uparrow}(G * H)=\left\{\vec{r} \in \mathbb{R}_{(\geq)}^{2} \mid \vec{r} \cdot \vec{a}, \vec{r} \cdot \vec{b} \geq 0\right\} \cup$

$$
\cup\left\{\vec{r} \in \mathbb{R}_{(\leq)}^{2} \mid \vec{r} \cdot \vec{a}, \vec{r} \cdot \vec{a}^{d}, \vec{r} \cdot \vec{b}, \vec{r} \cdot \vec{b}^{d} \geq 0\right\} .
$$

(4) $\mathcal{C}_{S O D}(G * H)=\left\{\vec{r} \in \mathbb{R}_{(\geq)}^{2} \mid \vec{r} \cdot \vec{a}=\vec{r} \cdot \vec{b}=0\right\} \cup$

$$
\cup\left\{\vec{r} \in \mathbb{R}_{(\leq)}^{2} \mid \vec{r} \cdot \vec{a}=\vec{r} \cdot \vec{a}^{d}=\vec{r} \cdot \vec{b}=\vec{r} \cdot \vec{b}^{d}=0\right\} .
$$

(5) $\mathcal{D}^{\uparrow}(G * H)=\left\{\vec{r} \in \mathbb{R}^{2} \mid \vec{r} \cdot \vec{a} \geq 0, \vec{r} \cdot \vec{b}^{d} \geq 0\right\}$.

(6) $\mathcal{C}(G * H)=\left\{\vec{r} \in \mathbb{R}^{2} \mid \vec{r} \cdot \vec{a}=\vec{r} \cdot \vec{b}^{d}=0\right\}$.

Proof. Set $F=G * H$,

(1) Let us assume that $\vec{r} \in \mathcal{D}_{\mathrm{OD}}^{\uparrow}(F)$. By Lemma 4.16 we can find $\mathbf{x} \in[0,1]_{(\geq)}^{2}$ and $c>0$ such that $\mathbf{x}+c \vec{r} \in[0,1]_{(\geq)}^{2}$, where $\mu+(\mathbf{x}+c \vec{r}) \cdot \vec{a}=G(\mathbf{x}+c \vec{r})=$ $F(\mathbf{x}+c \vec{r}) \geq F(\mathbf{x})=G(\mathbf{x})=\mu+\mathbf{x} \cdot \vec{a}$, hence $\vec{r} \cdot \vec{a} \geq 0$ and $\vec{r} \in \mathcal{D}_{\mathrm{OD}}^{\uparrow}(G)$. Equally we can find $\mathbf{x} \in[0,1]_{(\leq)}^{2}$ and $c>0$ with $\mathbf{x}+c \vec{r}_{\sigma} \in[0,1]_{(\leq)}^{2}$, from where $\vec{r} \in \mathcal{D}_{\mathrm{OD}}^{\uparrow}(H)$. Therefore, by Proposition 5.2 we have $\mathcal{D}_{\mathrm{OD}}^{\uparrow}(F)=\mathcal{D}_{\mathrm{OD}}^{\uparrow}(G) \cap \mathcal{D}_{\mathrm{OD}}^{\uparrow}(H)$.

(2) Proceed analogously as in (1).

(3) By Proposition 3.3. $\mathcal{D}_{\mathrm{SOD}}^{\uparrow}(F) \cap \mathbb{R}_{(\geq)}^{2}=\mathcal{D}_{\mathrm{OD}}^{\uparrow}(F) \cap \mathbb{R}_{(\geq)}^{2}$.

Let us assume that $\vec{r} \in \mathbb{R}_{(\leq)}^{2}$ satisfies $\vec{r} \cdot \vec{a}, \vec{r} \cdot \vec{b}, \vec{r} \cdot \vec{a}^{d}, \vec{r} \cdot \vec{b}^{d} \geq 0$. Let us see that $F$ is SOD $\vec{r}$-increasing. Let $\mathbf{x} \in[0,1]^{2}, c>0$ and $\sigma \in \mathcal{S}_{2}$ such that $\mathbf{x}_{\sigma} \in[0,1]_{(\geq)}^{2}, \mathbf{x}_{\sigma}+c \vec{r} \in[0,1]^{2}$.

Suppose first that $\sigma=\mathrm{id}$, then $\mathbf{x} \in[0,1]_{(\geq)}^{2}$. We must show that $F(\mathbf{x}+c \vec{r}) \geq$ 470 $F(\mathbf{x})$.

(a) If $\mathbf{x}+c \vec{r} \in[0,1]_{(\geq)}^{2}$, then $F(\mathbf{x}+c \vec{r})-F(\mathbf{x})=c \vec{r} \cdot \vec{a} \geq 0$. 
(b) If $\mathbf{x}+c \vec{r} \in[0,1]_{(<)}^{2}$, then by Lemma 5.3 there exists $c^{\prime} \in(0, c)$ such that $\mathbf{x}+c^{\prime} \vec{r} \in[0,1]_{(=)}^{2}$. Therefore, if we put $\mathbf{y}=\mathbf{x}+c^{\prime} \vec{r}$, then $\mathbf{y}+\left(c-c^{\prime}\right) \vec{r}=$ $\mathbf{x}+c \vec{r} \in[0,1]_{(\geq)}^{2}$. Thus, since $\mathbf{y} \in[0,1]_{(=)}^{2}$,

$$
\begin{aligned}
F(\mathbf{x}+c \vec{r})-F(\mathbf{x}) & =F(\mathbf{x}+c \vec{r})-F(\mathbf{y})+F(\mathbf{y})-F(\mathbf{x}) \\
& =F\left(\mathbf{y}+\left(c-c^{\prime}\right) \vec{r}\right)-F(\mathbf{y})+F\left(\mathbf{x}+c^{\prime} \vec{r}\right)-F(\mathbf{x}) \\
& =\left(c-c^{\prime}\right) \vec{r}^{d} \cdot \vec{b}+c^{\prime} \vec{r} \cdot \vec{a} \geq 0 .
\end{aligned}
$$

Suppose now that $\sigma=(12)$. We can assume that $\mathbf{x} \in[0,1]_{(<)}^{2}$ and so $F(\mathbf{x})=\mu+\mathbf{x}_{\sigma} \cdot \vec{b}$.

(c) If $\mathbf{x}_{\sigma}+c \vec{r} \in[0,1]_{(\geq)}^{2}$, then $F\left(\mathbf{x}+c \vec{r}_{\sigma}\right)-F(\mathbf{x})=c \vec{r} \cdot \vec{b} \geq 0$.

(d) If $\mathbf{x}_{\sigma}+c \vec{r} \in[0,1]_{(<)}^{2}$, then by Lemma 5.3 there exists $c^{\prime} \in(0, c)$ such that $\mathbf{x}+c^{\prime} \vec{r}_{\sigma} \in[0,1]_{(=)}^{2}$. Therefore, if we put $\mathbf{y}=\mathbf{x}+c^{\prime} \vec{r}_{\sigma}$, then $\mathbf{y}+\left(c-c^{\prime}\right) \vec{r}_{\sigma}=$ $\mathbf{x}+c \vec{r}_{\sigma} \in[0,1]_{(\geq)}^{2}$. Thus, since $\mathbf{y} \in[0,1]_{(=)}^{2}$,

$$
\begin{aligned}
F\left(\mathbf{x}+c \vec{r}_{\sigma}\right)-F(\mathbf{x}) & =F\left(\mathbf{x}+c \vec{r}_{\sigma}\right)-F(\mathbf{y})+F(\mathbf{y})-F(\mathbf{x}) \\
& =F\left(\mathbf{y}+\left(c-c^{\prime}\right) \vec{r}_{\sigma}\right)-F(\mathbf{y})+F\left(\mathbf{x}+c^{\prime} \vec{r}_{\sigma}\right)-F(\mathbf{x}) \\
& =\left(c-c^{\prime}\right) \vec{r}_{\sigma} \cdot \vec{a}+c^{\prime} \vec{r} \cdot \vec{b} \geq 0 .
\end{aligned}
$$

Therefore $F$ is SOD $\vec{r}$-increasing.

Let us assume now that $F$ is SOD $\vec{r}$-increasing, where $\vec{r} \in \mathbb{R}_{(\leq)}^{2}$. In particular, by Proposition $3.2, F$ is OD $\vec{r}$-increasing, hence $\vec{r} \cdot \vec{a}, \vec{r} \cdot \vec{b} \geq 0$ by 1 . It rests to show that if $r_{1}<r_{2}$ then $\vec{r} \cdot \vec{a}^{d}, \vec{r} \cdot \vec{b}^{d} \geq 0$. Indeed, from cases $(b)$ and $(d)$, the definition of SOD $\vec{r}$-increasingness for $\mathbf{y}$ ensures that $\vec{r} \cdot \vec{a}^{d}, \vec{r} \cdot \vec{b}^{d} \geq 0$.

(4) Proceed as in the preceding item with equalities instead of inequalities.

(5) Let $\vec{r} \in \mathcal{D}^{\uparrow}(F)$. By Lemma 5.3 , there exist $\mathbf{x} \in[0,1]_{(\geq)}^{2}$ and $c>0$ such that $\mathbf{x}+c \vec{r} \in[0,1]_{(\geq)}^{2}$. Thus, $0 \leq F(\mathbf{x}+c \vec{r})-F(\mathbf{x})=c \vec{r} \cdot \vec{a}$, so $\vec{r} \cdot \vec{a} \geq 0$. Similarly, by Lemma 5.3 , there exist $\mathbf{x} \in[0,1]_{(\leq)}^{2}$ and $c>0$ such that $\mathbf{x}+c \vec{r} \in$ $[0,1]_{(\leq)}^{2}$. Therefore, $0 \leq F(\mathbf{x}+c \vec{r})-F(\mathbf{x})=c \vec{r} \cdot \vec{b}^{d}$, so $\vec{r} \cdot \vec{b}^{d} \geq 0$.

Conversely, let $\vec{r} \in \mathbb{R}^{2}$ be a vector such that $\vec{r} \cdot \vec{a} \geq 0$ and $\vec{r} \cdot \vec{b}^{d} \geq 0$. In order to prove that $\vec{r} \in \mathcal{D}^{\uparrow}(F)$, let $\mathbf{x} \in[0,1]^{2}$ and $c>0$ such that $\mathbf{x}, \mathbf{x}+c \vec{r} \in[0,1]^{2}$. We have four cases.

- If $\mathbf{x}, \mathbf{x}+c \vec{r} \in[0,1]_{(\geq)}^{2}$, then $F(\mathbf{x}+c \vec{r})-F(\mathbf{x})=c \vec{r} \cdot \vec{a} \geq 0$.

- If $\mathbf{x}, \mathbf{x}+c \vec{r} \in[0,1]_{(\leq)}^{2}$, then $F(\mathbf{x}+c \vec{r})-F(\mathbf{x})=c \vec{r} \cdot \vec{b}^{d} \geq 0$.

- If $\mathbf{x} \in[0,1]_{(>)}^{2}$ and $\mathbf{x}+c \vec{r} \in[0,1]_{(<)}^{2}$, there is $c^{\prime} \in(0, c)$ such that $\mathbf{x}+c^{\prime} \vec{r} \in$ $[0,1]_{(=)}^{2}$. If $\mathbf{y}=\mathbf{x}+c^{\prime} \vec{r}$, then $\mathbf{y}+\left(c-c^{\prime}\right) \vec{r}=\mathbf{x}+c \vec{r}$. As $\mathbf{x}, \mathbf{y}=\mathbf{x}+c^{\prime} \vec{r} \in$ $[0,1]_{(\geq)}^{2}$ and $\mathbf{y}, \mathbf{y}+\left(c-c^{\prime}\right) \vec{r}=\mathbf{x}+c \vec{r} \in[0,1]_{(\leq)}^{2}$, then

$$
F(\mathbf{x}+c \vec{r})-F(\mathbf{x})=F(\mathbf{x}+c \vec{r})-F(\mathbf{y})+F(\mathbf{y})-F(\mathbf{x})
$$




$$
\begin{aligned}
& =F\left(\mathbf{y}+\left(c-c^{\prime}\right) \vec{r}\right)-F(\mathbf{y})+F\left(\mathbf{x}+c^{\prime} \vec{r}\right)-F(\mathbf{x}) \\
& =\left(c-c^{\prime}\right) \vec{r} \cdot \vec{a}+c^{\prime} \vec{r} \cdot \vec{b}^{d} \geq 0 .
\end{aligned}
$$

- If $\mathbf{x} \in[0,1]_{(<)}^{2}$ and $\mathbf{x}+c \vec{r} \in[0,1]_{(>)}^{2}$, there is $c^{\prime} \in(0, c)$ such that $\mathbf{x}+c^{\prime} \vec{r} \in$ $[0,1]_{(=)}^{2}$. If $\mathbf{y}=\mathbf{x}+c^{\prime} \vec{r}$, then $\mathbf{y}+\left(c-c^{\prime}\right) \vec{r}=\mathbf{x}+c \vec{r}$. As $\mathbf{x}, \mathbf{y}=\mathbf{x}+c^{\prime} \vec{r} \in$ $[0,1]_{(\leq)}^{2}$ and $\mathbf{y}, \mathbf{y}+\left(c-c^{\prime}\right) \vec{r}=\mathbf{x}+c \vec{r} \in[0,1]_{(\geq)}^{2}$, then

$$
\begin{aligned}
F(\mathbf{x}+c \vec{r})-F(\mathbf{x}) & =F(\mathbf{x}+c \vec{r})-F(\mathbf{y})+F(\mathbf{y})-F(\mathbf{x}) \\
& =F\left(\mathbf{y}+\left(c-c^{\prime}\right) \vec{r}\right)-F(\mathbf{y})+F\left(\mathbf{x}+c^{\prime} \vec{r}\right)-F(\mathbf{x}) \\
& =\left(c-c^{\prime}\right) \vec{r} \cdot \vec{b}^{d}+c^{\prime} \vec{r} \cdot \vec{a} \geq 0 .
\end{aligned}
$$

(6) Proceed as in the preceding item with equalities instead of inequalities.

Example 5.5. Consider the Eukasiewicz implication $\mathrm{I}_{L}:[0,1]^{2} \rightarrow[0,1]$ given by $\mathrm{I}_{L}(\mathbf{x})=\min \left\{1,1-x_{1}+x_{2}\right\}$ if $\mathbf{x}=\left(x_{1}, x_{2}\right) \in[0,1]^{2}$. Then we have

$$
\mathrm{I}_{L}=\mathrm{OL}[1,(-1,1)] * \mathrm{OL}[1,(0,0)]
$$

(by Corollary $4.12,[1,(-1,1)]$ and $[1,(0,0)]$ define ordered linear fusion functions; as $1=1$ and $-1+1=0+0$, we may consider its $(*)$-product by Lemma 495 5.3. As an application of Proposition 5.4 we have:

(1) $\mathcal{D}^{\uparrow}\left(\mathrm{I}_{L}\right)=\mathcal{D}_{\mathrm{OD}}^{\uparrow}\left(\mathrm{I}_{L}\right)=\left\{\vec{r} \in \mathbb{R}^{2} \mid r_{1} \leq r_{2}\right\}$.

(2) $\mathcal{D}_{\mathrm{SOD}}^{\uparrow}\left(\mathrm{I}_{L}\right)=\mathcal{C}\left(\mathrm{I}_{L}\right)=\mathcal{C}_{\mathrm{SOD}}\left(\mathrm{I}_{L}\right)=\mathcal{C}_{\mathrm{OD}}\left(\mathrm{I}_{L}\right)=\left\{\vec{r} \in \mathbb{R}^{2} \mid r_{1}=r_{2}\right\}$.

Definition 5.6. Set $A=\{1, \ldots, n\}$ and, if $\alpha \in \mathcal{S}_{n}, A^{\alpha}=\left(A_{1}^{\alpha}, \ldots, A_{n}^{\alpha}\right)$, where

$$
A_{i}^{\alpha}=\{\alpha(i), \alpha(i+1), \ldots, \alpha(n)\}=A \backslash\{\alpha(1), \ldots, \alpha(i-1)\}
$$

Let now $\mathfrak{m}: 2^{A} \rightarrow[0,1]$ be a fuzzy measure (that is, $\mathfrak{m}$ satisfies $\mathfrak{m}(\emptyset)=0$, $\mathfrak{m}(A)=1$, and $\mathfrak{m}(X) \leq \mathfrak{m}(Y)$ if $X, Y \in 2^{A}$ and $\left.X \subseteq Y\right)$. If $\left(X_{1}, \ldots, X_{n}\right) \in$ $\left(2^{A}\right)^{n}$, we put $\widetilde{\mathfrak{m}}\left(X_{1}, \ldots, X_{n}\right)=\left(\mathfrak{m}\left(X_{1}\right), \ldots, \mathfrak{m}\left(X_{n}\right)\right)$

We set

$$
C=\left(\begin{array}{ccccc}
1 & -1 & 0 & \ldots & 0 \\
0 & 1 & -1 & \ldots & 0 \\
& & \ddots & \ddots & \\
0 & 0 & \ldots & 1 & -1 \\
0 & 0 & \ldots & 0 & 1
\end{array}\right)
$$

for the $n \times n$ matrix obtained from the identity matrix $I_{n}$ by putting -1 above the main diagonal and $C^{\mathrm{T}}$ for its transpose. Observe that, if $\mathbf{x}, \mathbf{y} \in \mathbb{R}^{n}$, then $\mathrm{x} C \cdot \mathbf{y}=\mathbf{x} \cdot \mathbf{y} C^{\mathrm{T}}$. 
The discrete Choquet integral of $\mathbf{x} \in[0,1]^{n}$ with respect to $\mathfrak{m}$ is the function

$$
\mathrm{C}_{\mathfrak{m}}:[0,1]^{n} \rightarrow[0,1]
$$

given by, if $\mathbf{x} \in[0,1]^{n}$ and $\alpha \in \mathcal{S}_{n}$ is such that $\mathbf{x}_{\alpha} \in[0,1]_{(\leq)}^{n}$,

$$
\mathrm{C}_{\mathfrak{m}}(\mathbf{x})=\mathbf{x}_{\alpha} C \cdot \widetilde{\mathfrak{m}}\left(A^{\alpha}\right)=\mathbf{x}_{\alpha} \cdot \widetilde{\mathfrak{m}}\left(A^{\alpha}\right) C^{\mathrm{T}} .
$$

Proposition 5.7. Let $\mathfrak{m}: 2^{A} \rightarrow[0,1]$ be a fuzzy measure and consider the associated Choquet integral $\mathrm{C}_{\mathfrak{m}}:[0,1]^{n} \rightarrow[0,1]$.Then

$$
\begin{aligned}
\mathcal{D}_{O D}^{\uparrow}\left(\mathrm{C}_{\mathfrak{m}}\right) & =\left\{\vec{r} \in \mathbb{R}^{n} \mid \vec{r} \cdot \widetilde{\mathfrak{m}}\left(A^{\alpha}\right) C^{\mathrm{T}} \geq 0 \forall \alpha \in \mathcal{S}_{n}\right\}, \\
\mathcal{C}_{O D}\left(\mathrm{C}_{\mathfrak{m}}\right) & =\left\{\vec{r} \in \mathbb{R}^{n} \mid \vec{r} \cdot \widetilde{\mathfrak{m}}\left(A^{\alpha}\right) C^{\mathrm{T}}=0 \forall \alpha \in \mathcal{S}_{n}\right\} .
\end{aligned}
$$

Proof. Let $\mathbf{x} \in[0,1]^{n}, \alpha \in \mathcal{S}_{n}$ and $c \in \mathbb{R}^{+}$with $\mathbf{x}_{\alpha}, \mathbf{x}_{\alpha}+c \vec{r} \in[0,1]_{(\leq)}^{n}$. Then, with $\mathbf{y}=\mathbf{x}+c \vec{r}_{\alpha^{-1}}$, we have $\mathbf{y}_{\alpha}=\mathbf{x}_{\alpha}+c \vec{r} \in[0,1]_{(\leq)}^{n}$ and so

$$
\begin{aligned}
\mathrm{C}_{\mathfrak{m}}(\mathbf{y}) & =\mathbf{y}_{\alpha} \cdot \widetilde{\mathfrak{m}}\left(A^{\alpha}\right) C^{\mathrm{T}} \\
& =\mathbf{x}_{\alpha} \cdot \widetilde{\mathfrak{m}}\left(A^{\alpha}\right) C^{\mathrm{T}}+c \vec{r} \cdot \widetilde{\mathfrak{m}}\left(A^{\alpha}\right) C^{\mathrm{T}} \\
& =\mathrm{C}_{\mathfrak{m}}(\mathbf{x})+c\left(\vec{r} \cdot \widetilde{\mathfrak{m}}\left(A^{\alpha}\right) C^{\mathrm{T}}\right),
\end{aligned}
$$

whence the thesis.

Corollary 5.8. Let $\mathfrak{m}: 2^{A} \rightarrow[0,1]$ be a fuzzy measure. Then the associated Choquet integral $\mathrm{C}_{\mathfrak{m}}:[0,1]^{n} \rightarrow[0,1]$ is SOD $\vec{r}$-increasing for each $\vec{r} \in \mathbb{R}^{n}$ such that $r_{i} \geq 0,1 \leq i \leq n$.

Proof. The $i$-th term of $\widetilde{\mathfrak{m}}\left(A^{\alpha}\right) C^{\mathrm{T}}$, with the convention $\mathfrak{m}\left(A_{n+1}^{\alpha}\right)=0$, is $\mathfrak{m}\left(A_{i}^{\alpha}\right)-$ $\mathfrak{m}\left(A_{i+1}^{\alpha}\right) \geq 0$ as $\mathfrak{m}$ is a fuzzy measure, whereby the thesis.

Let us consider the Choquet integral for $n=2$ in some detail. A fuzzy measure $\mathfrak{m}:\{1,2\}^{2} \rightarrow L$ corresponds to

$$
\left(\begin{array}{cccc}
\emptyset & \{1\} & \{2\} & \{1,2\} \\
0 & q_{1} & q_{2} & 1
\end{array}\right),
$$

where $0 \leq q_{1}, q_{2} \leq 1$, so that $\mathfrak{m}$ is totally determined by the pair $\left(q_{1}, q_{2}\right)$. We set $\mathfrak{m} \equiv\left(q_{1}, q_{2}\right)$. We have

$$
\widetilde{\mathfrak{m}}\left(A^{\alpha}\right) C^{\mathrm{T}}=\left\{\begin{aligned}
\left(1, q_{2}\right)\left(\begin{array}{cc}
1 & 0 \\
-1 & 1
\end{array}\right) & =\left(1-q_{2}, q_{2}\right) \text { if } \alpha=\mathrm{id}, \\
\left(1, q_{1}\right)\left(\begin{array}{cc}
1 & 0 \\
-1 & 1
\end{array}\right) & =\left(1-q_{1}, q_{1}\right) \text { if } \alpha=(12) .
\end{aligned}\right.
$$


And the conditions of Proposition $5.7 \vec{r} \cdot \widetilde{\mathfrak{m}}\left(A^{\alpha}\right) C^{\mathrm{T}} \geq 0 \forall \alpha \in S_{2}$ translate here to

$$
\left\{\begin{array}{l}
\left(r_{1}, r_{2}\right) \cdot\left(1-q_{1}, q_{1}\right) \geq 0, \\
\left(r_{1}, r_{2}\right) \cdot\left(1-q_{2}, q_{2}\right) \geq 0 .
\end{array}\right.
$$

This corresponds in the plane $\mathbb{R}^{2}$ to the intersection of the semiplanes rightwards and/or upwards the borders $\left(1-q_{i}\right) x_{1}+q_{i} x_{2}=0, i=1$, 2. For instance, the case $\mathfrak{m} \equiv(1 / 2,1 / 8)$ is shown in Figure 6 .

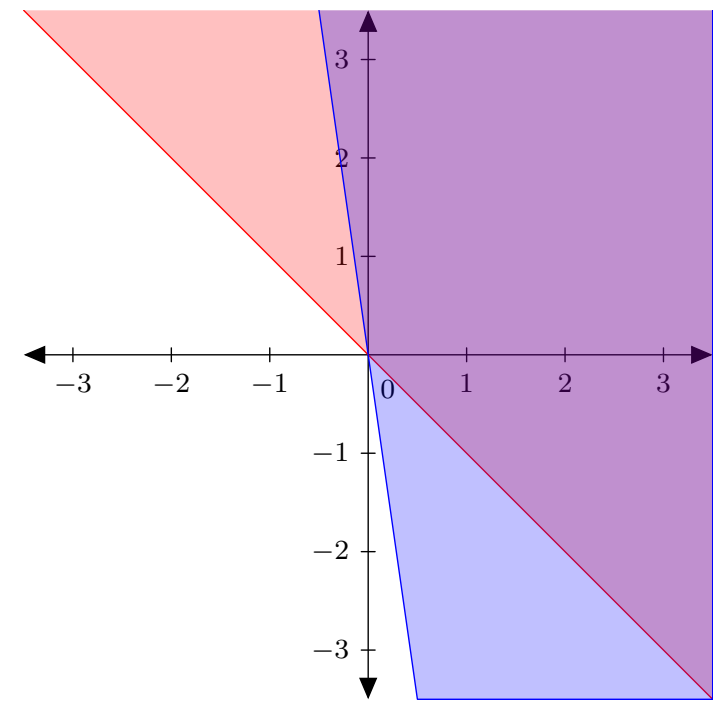

Figure 6: Intersection of the semiplanes from the conditions in 11 for the case $\mathfrak{m} \equiv(1 / 2,1 / 8)$.

Clearly, $\mathcal{C}_{\mathrm{OD}}(1 / 2,1 / 2)$ corresponds to the line $x_{1}+x_{2}=0$. If $q_{1} \neq q_{2}$, then $\mathcal{C}_{\mathrm{OD}}\left(q_{1}, q_{2}\right)=\{(0,0)\}$.

Let us set $\mathbf{q}_{i}=\left(1-q_{i}, q_{i}\right), i=1$, 2. So $\vec{r} \cdot \widetilde{\mathfrak{m}}\left(A^{\alpha}\right) C^{\mathrm{T}} \geq 0$ is $\vec{r} \cdot \mathbf{q}_{i} \geq 0, i=1,2$.

Observe that

$$
\mathrm{C}_{\left(q_{1}, q_{2}\right)} \mathbf{x}= \begin{cases}\mathbf{x} \cdot \mathbf{q}_{2} & \text { if } x_{1} \leq x_{2} \\ \mathbf{x} \cdot \mathbf{q}_{1} & \text { if } x_{1} \geq x_{2}\end{cases}
$$

Example 5.9. Let $\mu \in[0,1), \eta \in[0,1-\mu], q_{1}, q_{2} \in[0, \eta]$ and set $\mathbf{q}_{i}=\left(1-q_{i}, q_{i}\right)$, $i=1,2$. For the corresponding Choquet integral we have

$$
\mu+\eta \mathrm{C}_{\left(q_{1}, q_{2}\right)}=\mathrm{OL}\left[\mu, \eta \mathbf{q}_{1}\right] * \mathrm{OL}\left[\mu, \eta \mathbf{q}_{2}\right]
$$


(by Corollary 4.12 , each $\left[\mu, \eta\left(1-q_{i}, q_{i}\right)\right]$ defines an ordered linear fusion function, $i=1,2$; and as $\mu=\mu$ and $\eta-\eta q_{1}+\eta q_{1}=\eta-\eta q_{2}+\eta q_{2}$, we may consider its

(*)-product by Lemma 5.3. As a direct application of Proposition 5.4

(1) $\mathcal{D}_{O D}^{\uparrow}\left(\mu+\eta \mathrm{C}_{\left(q_{1}, q_{2}\right)}\right)=\left\{\vec{r} \in \mathbb{R}^{2} \mid \eta \vec{r} \cdot\left(1-q_{i}, q_{i}\right) \geq 0, i=1,2\right\}$.

(2) $\mathcal{C}_{O D}\left(\mu+\eta \mathrm{C}_{\left(q_{1}, q_{2}\right)}\right)=\left\{\vec{r} \in \mathbb{R}^{2} \mid \eta \vec{r} \cdot\left(1-q_{i}, q_{i}\right)=0, i=1,2\right\}$.

(3) $\mathcal{D}_{S O D}^{\uparrow}\left(\mu+\eta \mathrm{C}_{\left(q_{1}, q_{2}\right)}\right)=\left\{\vec{r} \in \mathbb{R}_{(\geq)}^{2} \mid \eta \vec{r} \cdot\left(1-q_{i}, q_{i}\right) \geq 0, i=1,2,\right\} \cup$ $\cup\left\{\vec{r} \in \mathbb{R}_{(\leq)}^{2} \mid \eta \vec{r} \cdot\left(1-q_{i}, q_{i}\right) \geq 0, \eta \vec{r} \cdot\left(q_{i}, 1-q_{i}\right) \geq 0, i=1,2\right\}$.

(4) $\mathcal{C}_{S O D}\left(\mu+\eta \mathrm{C}_{\left(q_{1}, q_{2}\right)}\right)=\left\{\vec{r} \in \mathbb{R}_{(\geq)}^{2} \mid \eta \vec{r} \cdot\left(1-q_{i}, q_{i}\right)=0, i=1,2\right\} \cup$ $\cup\left\{\vec{r} \in \mathbb{R}_{(\leq)}^{2} \mid \eta \vec{r} \cdot\left(1-q_{i}, q_{i}\right)=\eta \vec{r} \cdot\left(q_{i}, 1-q_{i}\right)=0, i=1,2\right\}$.

(5) $\mathcal{D}^{\uparrow}\left(\mu+\eta \mathrm{C}_{\left(q_{1}, q_{2}\right)}\right)=\left\{\vec{r} \in \mathbb{R}^{2} \mid \eta \vec{r} \cdot\left(1-q_{1}, q_{1}\right) \geq 0, \eta \vec{r} \cdot\left(q_{2}, 1-q_{2}\right) \geq 0\right\}$.

(6) $\mathcal{C}\left(\mu+\eta \mathrm{C}_{\left(q_{1}, q_{2}\right)}\right)=\left\{\vec{r} \in \mathbb{R}^{2} \mid \eta \vec{r} \cdot\left(1-q_{1}, q_{1}\right)=\eta \vec{r} \cdot\left(q_{2}, 1-q_{2}\right)=0\right\}$.

For $\mu=0$ and $\eta=1$ we get the Choquet integral and let us observe that $\mathrm{OL}\left[0, \mathbf{q}_{i}\right]$ is an OWA operator, $i=1,2$.

\section{Remark 5.10.}

(1) Let $\mathbf{x}=\left(x_{1}, x_{2}\right) \in[0,1]^{2}$ and $\vec{r}=\left(r_{1}, r_{2}\right) \in \mathbb{R}^{2}$.

Let $M:[0,1]^{2} \rightarrow[0,1]$ be given by $M(\mathbf{x})=\left|x_{1}-x_{2}\right|$. Then, $M$ is OD $\vec{r}-$ increasing if and only if $r_{1} \geq r_{2}$ (see Corollary 4.21). We have that $I_{M}^{0}(\mathbf{x})=$ $\max \left\{0, x_{1}-x_{2}\right\}$ defines an $\vec{r}$-increasing function such that $\widehat{I_{M}^{0}}=M$.

Consider now $M^{c}:[0,1]^{2} \rightarrow[0,1]$, that is $M^{c}(\mathbf{x})=1-\left|x_{1}-x_{2}\right|=1-$ $\max \left(x_{1}, x_{2}\right)+\min \left(x_{1}, x_{2}\right)$, an OD $\vec{r}$-increasing function if and only if $r_{1} \leq r_{2}$ (see Proposition 4.22). Then $I_{M^{c}}^{1}=I_{L}$ (the Lukasiewicz implication) is $\vec{r}$ increasing and $\widehat{I_{L}}=M^{c}$.

As $\widehat{I_{L}}=M^{c}$ we can deduce that in the hypothesis of Proposition 3.10 it is not true in general that $\widehat{F}$ is SOD $\vec{r}$-increasing: take $\vec{r}=\left(r_{1}, r_{2}\right)$ with $r_{1}<r_{2}$; then $\mathrm{I}_{L}$ is $\vec{r}$-increasing by Example 5.5 but $\widehat{I_{L}}$ is not SOD $\vec{r}$ increasing by Proposition 4.22 .

(2) Let $M:[0,1]^{2} \rightarrow[0,1]$ as in (1). We have seen in Corollary 4.21 and Proposition 4.22 that $\mathcal{D}_{\text {SOD }}^{\uparrow}(M)=\left\{\vec{r} \in \mathbb{R}^{2} \mid r_{1} \geq r_{2}\right\}$ and $\mathcal{D}_{\text {SOD }}^{\uparrow}\left(M^{c}\right)=\left\{\vec{r} \in \mathbb{R}^{2} \mid\right.$ $\left.r_{1}=r_{2}\right\}$, hence Proposition 3.5(2) is not true for T = SOD. And taking into account the proof of Proposition 3.5(2), neither is valid Proposition 3.4 (3) for $\mathrm{T}=\mathrm{SOD}$. 


\section{General properties}

550 monotonicity in terms of directional, SOD and OD monotonicity. Thus, they are a link between the different notions of monotonicity. Additionally, we extend a general property of OD monotone functions from [5] to the case of SOD monotone functions. We finish the section with two results on how OD and

(3) $F$ is SOD $\vec{e}_{i}$-increasing $\forall i \in\{1, \ldots, n\}$.

(4) $F$ is $O D \vec{e}_{i}$-increasing $\forall i \in\{1, \ldots, n\}$.

Proof. It is obvious that (1) $\Longleftrightarrow(2)$, and (1) $\Longrightarrow(3)$ as $\mathbf{x} \leq \mathbf{x}+c\left(\vec{e}_{i}\right)_{\sigma^{-1}}$ for all $c>0$ and $\sigma \in \mathcal{S}_{n}$ ).

565

$(3) \Longrightarrow(1)$ Let $\mathbf{x}, \mathbf{y} \in[0,1]^{n}$ such that $\mathbf{x} \leq \mathbf{y}$. We must show that $F(\mathbf{x}) \leq F(\mathbf{y})$ and so we may assume that $\mathbf{x}<\mathbf{y}$. Let $\sigma \in \mathcal{S}_{n}$ be such that $x_{\sigma} \in[0,1]_{(\geq)}^{n}$. Observe that $\mathbf{x}<\mathbf{y}$ is equivalent to $\mathbf{x}_{\sigma}<\mathbf{y}_{\sigma}$. Let $i=\max \{j \in$ $\left.\{1, \ldots, n\} \mid x_{\sigma(j)}<y_{\sigma(j)}\right\}$.

So we have the following scheme (it could be $i=n$ ):

$$
\begin{array}{ccccccccc}
\left(\mathbf{x}_{\sigma}\right): & x_{\sigma(1)} \geq & \ldots & \geq x_{\sigma(i-1)} \geq & x_{\sigma(i)} \geq & x_{\sigma(i+1)} \geq & \ldots & \geq x_{\sigma(n)} \\
& \mid \wedge & \ldots & \mid \wedge & \wedge & \| & \ldots & \| \\
\left(\mathbf{y}_{\sigma}\right): & y_{\sigma(1)} & \ldots & y_{\sigma(i-1)} & y_{\sigma(i)} & y_{\sigma(i+1)} & \ldots & y_{\sigma(n)}
\end{array}
$$

Let $c=y_{\sigma(i)}-x_{\sigma(i)}$. We have

$$
\begin{aligned}
\mathbf{x}_{\sigma}+c \vec{e}_{i} & =\left(x_{\sigma(1)}, \ldots, x_{\sigma(i-1)}, x_{\sigma(i)}+c, x_{\sigma(i+1)}, \ldots, x_{\sigma(n)}\right) \\
& =\left(x_{\sigma(1)}, \ldots, x_{\sigma(i-1)}, y_{\sigma(i)}, y_{\sigma(i+1)}, \ldots, y_{\sigma(n)}\right) \in[0,1]^{n} .
\end{aligned}
$$

Hence, as $F$ is SOD $\vec{e}_{i}$-increasing, $F(\mathbf{x}) \leq F(\mathbf{z})$, where $\mathbf{z}=\mathbf{x}+c\left(\vec{e}_{i}\right)_{\sigma^{-1}}$. We have 570 $\mathbf{z}=\mathbf{x}+c \vec{e}_{j}=\left(x_{1}, \ldots, x_{j}+c, \ldots, x_{n}\right)=\left(x_{1}, \ldots, y_{j}, \ldots, x_{n}\right)$, where $j=\sigma(i)$.

Set now, if $\mathbf{v}=\left(v_{1}, \ldots, v_{n}\right), \mathbf{w}=\left(w_{1}, \ldots, w_{n}\right) \in[0,1]^{n}$ and $\mathbf{v} \leq \mathbf{w}$,

$$
\mathrm{d}(\mathbf{v}, \mathbf{w})=\left|\left\{k \mid v_{k}<w_{k}\right\}\right| .
$$

We have that $\mathbf{z} \leq \mathbf{y}$ and $\mathrm{d}(\mathbf{z}, \mathbf{y})=\mathrm{d}(\mathbf{x}, \mathbf{y})-1$, hence, if we reiterate the process, we conclude $F(\mathbf{x}) \leq F(\mathbf{z}) \leq \cdots \leq F(\mathbf{y})$, as required. 
(3) $\Longleftrightarrow$ (4) By the definitions it suffices to show (4) $\Longrightarrow$ (3). Let $\mathbf{x} \in[0,1]^{n}, \sigma \in \mathcal{S}_{n}, c>0$ and $i \in\{1, \ldots, n\}$ such that $\mathbf{x}_{\sigma} \in[0,1]_{(\geq)}^{n}$ and $\mathbf{x}_{\sigma}+c \vec{e}_{i} \in[0,1]^{n}$. Let us see that $F(\mathbf{x}) \leq F\left(\mathbf{x}+c\left(\vec{e}_{i}\right)_{\sigma^{-1}}\right)$.

Proceed by induction. If $i=1$, then $\mathbf{x}_{\sigma} \in[0,1]_{(\geq)}^{n}$ implies $\mathbf{x}_{\sigma}+c \vec{e}_{1} \in[0,1]_{(\geq)}^{n}$ and the thesis follows as $F$ is OD $\vec{e}_{1}$-increasing.

Assume that the result is true for $i-1$, where $2 \leq i \leq n$.

If $\mathbf{x}_{\sigma(i-1)} \geq \mathbf{x}_{\sigma(i)}+c$, then $\mathbf{x}_{\sigma}+c \vec{e}_{i} \in[0,1]_{(\geq)}^{n}$ and the thesis follows as $F$ is OD $\vec{e}_{i}$-increasing. Assume that $\mathbf{x}_{\sigma(i-1)}<\mathbf{x}_{\sigma(i)}+c$.

Set $t=x_{\sigma(i-1)}-x_{\sigma(i)}$. We have

$$
\mathbf{x}_{\sigma}+t \vec{e}_{i}=\left(x_{\sigma(1)}, \ldots, x_{\sigma(i-1)}^{i-1}, x_{\sigma(i-1)}^{i}, x_{\sigma(i+1)}, \ldots\right) \in[0,1]_{(\geq)}^{n},
$$

hence $F(\mathbf{x}) \leq F\left(\mathbf{x}+t\left(\vec{e}_{i}\right)_{\sigma^{-1}}\right)$ as $F$ is OD $\vec{e}_{i^{-}}$-increasing.

Set $\mathbf{y}=\mathbf{x}+t\left(\vec{e}_{i}\right)_{\sigma^{-1}}$, so $F(\mathbf{x}) \leq F(\mathbf{y})$.

Consider the transposition $\tau=(i-1 i)$. Observe that $\mathbf{y}_{(\sigma \tau)}=\left(\mathbf{y}_{\sigma}\right)_{\tau}=\mathbf{y}_{\sigma}$. Set $s=c-t$. As $x_{\sigma(i-1)}+c-t=x_{\sigma(i)}+c$ we have

$$
\mathbf{y}_{(\sigma \tau)}+s \vec{e}_{i-1}=\left(x_{\sigma(1)}, \ldots, x_{\sigma(i)}^{i-1}+c, x_{\sigma(i-1)}, x_{\sigma(i+1)}, \ldots\right) \in[0,1]^{n} .
$$

By the induction hypothesis, $F$ is OD $\vec{e}_{i-1}$-increasing, hence

$$
F(\mathbf{y}) \leq F\left(\mathbf{y}+s\left(\vec{e}_{i-1}\right)_{(\sigma \tau)^{-1}}\right) .
$$

As

$$
\begin{gathered}
\mathbf{y}+s\left(\vec{e}_{i-1}\right)_{(\sigma \tau)^{-1}}=\mathbf{y}+s\left(\vec{e}_{i-1}\right)_{\left(\tau \sigma^{-1}\right)}=\mathbf{y}+s\left(\vec{e}_{i}\right)_{\sigma^{-1}}= \\
=\mathbf{x}+(t+s)\left(\vec{e}_{i}\right)_{\sigma^{-1}}=\mathbf{x}+c\left(\vec{e}_{i}\right)_{\sigma^{-1}}
\end{gathered}
$$

we have $F(\mathbf{x}) \leq F(\mathbf{y}) \leq F\left(\mathbf{x}+c\left(\vec{e}_{i}\right)_{\sigma^{-1}}\right)$ and the proof is finished.

Corollary 6.2. Let $F:[0,1]^{n} \rightarrow[0,1]$ be a function. Then the following assertions are equivalent:

(1) F is increasing.

(2) $F$ is SOD $\vec{r}$-increasing for each $\vec{r} \in[0, \infty)^{n}$.

(3) $F$ is $O D \vec{r}$-increasing for each $\vec{r} \in[0, \infty)^{n}$.

Recall that a function $F:[0,1]^{n} \rightarrow[0,1]$ is said to be weakly increasing if $\lambda \in \mathbb{R}$ and $\left(x_{1}, \ldots, x_{n}\right),\left(x_{1}+\lambda, \ldots, x_{n}+\lambda\right) \in[0,1]^{n}$ implies $F\left(x_{1}, \ldots, x_{n}\right) \leq$ $F\left(x_{1}+\lambda, \ldots, x_{n}+\lambda\right)$.

Proposition 6.3. Let $F:[0,1]^{n} \rightarrow[0,1]$ and $\overrightarrow{1}=(1, \ldots, 1) \in \mathbb{R}^{n}$. The following assertions are equivalent. 
(1) $F$ is weakly increasing.

595

(2) F is $\overrightarrow{1}$-increasing.

(3) F is SOD $\overrightarrow{1}$-increasing.

(4) $F$ is $O D \overrightarrow{1}$-increasing.

Proof. The fact that $(1) \Longleftrightarrow(2)$ is simply the definition of weakly increasingness and, by Proposition 3.3 , (3) $\Longleftrightarrow$ (4).

$(2) \Longrightarrow(3)$ Let $\mathbf{x} \in[0,1], \sigma \in \mathcal{S}_{n}$ and $c \in \mathbb{R}^{+}$such that $\mathbf{x}_{\sigma} \in[0,1]_{(\geq)}^{n}$ and $\mathbf{x}_{\sigma}+c \vec{r} \in[0,1]^{n}$. Then $F\left(\mathbf{x}+c \overrightarrow{1}_{\sigma^{-1}}\right)=F(\mathbf{x}+c \overrightarrow{1}) \geq F(\mathbf{x})$ as $F$ is $\overrightarrow{1}$-increasing.

(3) $\Longrightarrow(2)$ Let $\mathbf{x}=\left(x_{1}, \ldots, x_{n}\right) \in[0,1]^{n}$ and $c \in \mathbb{R}^{+}$such that $\mathbf{x}+c \overrightarrow{1} \in$ $[0,1]^{n}$. Take $\sigma \in \mathcal{S}_{n}$ such that $\mathbf{x}_{\sigma} \in[0,1]_{(\geq)}^{n}$. We have that

$$
\mathbf{x}_{\sigma}+c \overrightarrow{1}=\mathbf{x}_{\sigma}+c \overrightarrow{1}_{\sigma}=(\mathbf{x}+c \overrightarrow{1})_{\sigma} \in[0,1]^{n}
$$

as $\mathbf{x}+c \vec{r} \in[0,1]^{n}$. So, by hypothesis,

$$
F(\mathbf{x}) \leq F\left(\mathbf{x}+c \overrightarrow{1}_{\sigma^{-1}}\right)=F(\mathbf{x}+c \overrightarrow{1}),
$$

and $F$ is $\overrightarrow{1}$-increasing.

The following theorem (see [5]) deals with OD increasingness along the linear combination of two directions.

Theorem 6.4 ([5]). Let $\vec{r}, \vec{s} \in \mathbb{R}^{n}$ and $a, b \in \mathbb{R}^{+}$. Let us assume that if $\mathbf{x} \in$ $[0,1]^{n}, c \in \mathbb{R}^{+}, \sigma \in \mathcal{S}_{n}$, then

$$
\begin{aligned}
\mathbf{x}_{\sigma}, \mathbf{x}_{\sigma}+c(a \vec{r}+b \vec{s}) & \in[0,1]_{(\geq)}^{n} \\
& \Longrightarrow \text { either } \mathbf{x}_{\sigma}+c a \vec{r} \in[0,1]_{(\geq)}^{n} \text { or } \mathbf{x}_{\sigma}+\operatorname{cbs} \in[0,1]_{(\geq)}^{n}
\end{aligned}
$$

Then if a function is both $O D \vec{r}$ - and $O D \vec{s}$-increasing then it is also $O D(a \vec{r}+b \vec{s})-$ increasing.

We present its extension to the case of SOD monotone functions. The proof is straightforward.

Theorem 6.5. Let $\vec{r}, \vec{s} \in \mathbb{R}^{n}$ and $a, b \in \mathbb{R}^{+}$. Let us assume that if $\mathbf{x} \in[0,1]^{n}$, $c \in \mathbb{R}^{+}, \sigma \in \mathcal{S}_{n}$, then

$$
\begin{aligned}
\mathbf{x}_{\sigma} \in[0,1]_{(\geq)}^{n}, \mathbf{x}_{\sigma}+ & c(a \vec{r}+b \vec{s}) \in[0,1]^{n} \\
& \Longrightarrow \text { either } \mathbf{x}_{\sigma}+\text { car } \in[0,1]^{n} \text { or } \mathbf{x}_{\sigma}+c b \vec{s} \in[0,1]^{n}
\end{aligned}
$$

Then if a function is both SOD $\vec{r}$ - and SOD $\vec{s}$-increasing then it is also SOD 
Finally, the next theorem shows a construction method of OD and SOD monotone functions, based on aggregating functions with the same type of monotonicity.

Theorem 6.6. Let $\vec{r} \in \mathbb{R}^{n}$, let $A:[0,1]^{n} \rightarrow[0,1]$ be an aggregation function and $F_{i}:[0,1]^{n} \rightarrow[0,1], 1 \leq i \leq m$, functions. Define

$$
A\left(F_{1}, \ldots, F_{m}\right):[0,1]^{n} \rightarrow[0,1]
$$

by $A\left(F_{1}, \ldots, F_{m}\right)(\mathbf{x})=A\left(F_{1}(\mathbf{x}), \ldots, F_{m}(\mathbf{x})\right)$. Then

(1) If $F_{i}$ is $O D \vec{r}$-increasing $\forall i \in\{1, \ldots, m\}$, then $A\left(F_{1}, \ldots, F_{m}\right)$ is also $O D$ $\vec{r}$-increasing.

(2) If $F_{i}$ is $S O D \vec{r}$-increasing $\forall i \in\{1, \ldots, m\}$, then $A\left(F_{1}, \ldots, F_{m}\right)$ is also $S O D$ $\vec{r}$-increasing.

Proof. It is straightforward.

As a consequence, the sets of all SOD $\vec{r}$-increasing functions and of all OD $\vec{r}$-increasing functions for a given $\vec{r} \in \mathbb{R}^{n}$ are convex.

Corollary 6.7. Let $\lambda_{1}, \ldots, \lambda_{n} \in[0,1]$ be such that $\lambda_{1}+\cdots+\lambda_{n}=1$. Let $T \in\{S O D, O D\}$ and $\vec{r} \in \mathbb{R}^{n}$. Then, if $F_{i}:[0,1]^{n} \rightarrow[0,1], 1 \leq i \leq n$, are $T$ $\vec{r}$-increasing functions, then their convex combination $\lambda_{1} F_{1}+\cdots+\lambda_{n} F_{n}$ is also $T \vec{r}$-increasing.

Proof. The result follows from Theorem 6.6, as $A\left(x_{1}, \ldots, x_{n}\right)=\lambda_{1} x_{1}+\cdots+$ $\lambda_{n} x_{n}$ defines an aggregation function.

\section{Conclusions}

We have defined the concept of strengthened ordered directional (SOD) monotonicity and studied some properties of the functions that are SOD monotone. We have also studied the relation between three notions of weaker forms of monotonicity, that of directional monotonicity, ordered directional monotonicity and strengthened ordered directional monotonicity. Additionally, we have introduced the family of linear fusion functions, the family of ordered linear fusion functions and an operation between functions that recover Choquet integrals and the Eukasiewicz implication. 


\section{Acknowledgements}

This work is partially supported by the grants APVV-14-0013 and TIN201677356-P (AEI/FEDER, UE).

640

\section{References}

[1] G. Beliakov, H. Bustince, and T. Calvo. A Practical Guide to Averaging Functions. Studies in Fuzziness and Soft Computing. Springer International Publishing, 2016.

[2] G. Beliakov, T. Calvo, and T. Wilkin. Three types of monotonicity of

[8] J. García-Lapresta and M. Martínez-Panero. Positional voting rules generated by aggregation functions and the role of duplication. International Journal of Intelligent Systems, 32(9):926-946, 2017. 
[9] M. Grabisch, J. Marichal, R. Mesiar, and E. Pap. Aggregation functions. Cambridge University Press, 2009.

[10] G. Lucca, J. Sanz, G. Dimuro, B. Bedregal, M. J. Asiain, M. Elkano, and H. Bustince. CC-integrals: Choquet-like copula-based aggregation functions and its application in fuzzy rule-based classification systems. Knowledge-Based Systems, 119:32 - 43, 2017.

[11] G. Lucca, J. A. Sanz, G. P. Dimuro, B. Bedregal, H. Bustince, and R. Mesiar. CF-Integrals: a new family of pre-aggregation functions with application to fuzzy rule-based classification systems. Information Sciences, 435:94-110, 2017.

[12] G. Lucca, J. A. Sanz, G. P. Dimuro, B. Bedregal, R. Mesiar, A. Kolesárová, and H. Bustince. Preaggregation functions: Construction and an application. IEEE Transactions on Fuzzy Systems, 24(2):260-272, 2016.

[13] R. Mesiar, A. Kolesárová, and A. Stupňanová. Quo vadis aggregation? International Journal of General Systems, 47(2):97-117, 2018.

[14] D. Paternain, J. Fernandez, H. Bustince, R. Mesiar, and G. Beliakov. Construction of image reduction operators using averaging aggregation functions. Fuzzy Sets and Systems, 261:87 - 111, 2015.

[15] M. Sesma-Sara, H. Bustince, E. Barrenechea, J. Lafuente, A. Kolsesárová, and R. Mesiar. Edge detection based on ordered directionally monotone functions. In Advances in Fuzzy Logic and Technology 2017, pages 301-307. Springer International Publishing, 2018.

[16] T. Wilkin and G. Beliakov. Weakly monotonic averaging functions. International Journal of Intelligent Systems, 30(2):144-169, 2015.

[17] R. R. Yager. On ordered weighted averaging aggregation operators in multicriteria decisionmaking. IEEE Transactions on Systems, Man, and Cybernetics, 18(1):183-190, 1988. 